Hamburger Beiträge zur Mathematik Nr. 336

July 2009

\title{
THE THREE-DIMENSIONAL ORIGIN OF THE CLASSIFYING ALGEBRA
}

\author{
Jürgen Fuchs $^{a}$, Christoph Schweigert ${ }^{b}$, Carl Stigner ${ }^{a}$ \\ a Teoretisk fysik, Karlstads Universitet \\ Universitetsgatan 21, S-65188 Karlstad \\ ${ }^{b}$ Organisationseinheit Mathematik, Universität Hamburg \\ Bereich Algebra und Zahlentheorie \\ Bundesstraße 55, D-20146 Hamburg
}

\begin{abstract}
It is known that reflection coefficients for bulk fields of a rational conformal field theory in the presence of an elementary boundary condition can be obtained as representation matrices of irreducible representations of the classifying algebra, a semisimple commutative associative complex algebra.

We show how this algebra arises naturally from the three-dimensional geometry of factorization of correlators of bulk fields on the disk. This allows us to derive explicit expressions for the structure constants of the classifying algebra as invariants of ribbon graphs in the three-manifold $S^{2} \times S^{1}$. Our result unravels a precise relation between intertwiners of the action of the mapping class group on spaces of conformal blocks and boundary conditions in rational conformal field theories.
\end{abstract}




\section{Introduction}

Structure constants of operator product expansions (OPEs) have played an important role in shaping our understanding of correlation functions in two-dimensional conformal field theory. In fact, one approach to conformal field theory has been to identify a subset of fundamental correlators - which are ultimately encoded in appropriate OPEs - from which all other correlators can be obtained by sewing. Since a given correlation function can typically be constructed from the fundamental correlators by sewing in several distinct ways, the uniqueness of the correlators imposes various necessary conditions on the sewing procedure. These conditions are known as sewing constraints, or factorization constraints [1,2, 3]. There are actually two different types of factorizations:

- Boundary factorization, involving a cutting of the world sheet along an interval that connects two points on its boundary, yields a correlator with two additional insertions of boundary fields.

- Bulk factorization, for which the cutting is along a circle in the interior of the world sheet, yields a correlator with two additional bulk field insertions.

In the guise of associativity of the OPE, constraints from bulk factorization have been central in the understanding of the OPE of bulk fields [4. Much later it was realized [5, 6] that the constraints on structure constants for boundary fields preserving a given boundary condition have in fact a simpler structure and give rise to an associative algebra in the tensor category of chiral data.

The OPE coefficients of bulk fields in the presence of a boundary are amenable as well. The one-point correlators of bulk fields on a disk, which may be collected in so-called boundary states, contain significant information of much interest for applications, like ground state degeneracies [7] or Ramond-Ramond charges of string compactifications [8]. Moreover, they provide essential information about annulus partition functions and thus encode the spectrum of boundary fields. Based on an analysis of specific classes of models, it was found that the reflection coefficients for bulk fields in the presence of an elementary boundary condition can be formulated in terms of representation matrices of irreducible representations of a classifying algebra [3, 9. 11 In particular, the elementary boundary conditions are in bijection with the irreducible representations of the classifying algebra. Further, there is evidence that the structure constants of the classifying algebra can be expressed in terms of traces of intertwiners for the mapping class group action on spaces of conformal blocks on the sphere [11, whereby they are related to the subbundle structure of the bundles of conformal blocks [12. These ideas have lead to concrete formulas for operator product coefficients [13, 14, 15, 16, 17] with a wide range of uses; see e.g. [18, 19, 20, 21] for some applications in string theory.

More recently, the TFT approach to the correlation functions of rational conformal field theories has provided a much more satisfactory understanding of RCFT correlators. The main idea of this approach can be summarized as follows. The chiral data of a conformal field theory are described by the structure of a modular tensor category [22,23], and a full local conformal field theory based on these chiral data corresponds to (a Morita class of) a symmetric special Frobenius algebra in that category $24,25,26,27,28$. A modular tensor category also gives rise to a three-dimensional topological field theory [29]. The TFT approach uses this topological field theory to construct the correlators of the local conformal field theory as invariants of ribbon graphs in three-manifolds with boundary. It has been shown [30] that the so obtained correlators are invariant under the mapping class group and obey all factorization constraints.

\footnotetext{
${ }^{1}$ The same algebra had arisen in the study of integrable lattice models [10].
} 
The purpose of this paper is to establish the existence of a classifying algebra for any RCFT - an associative commutative algebra $\mathcal{A}$ over the complex numbers with the property that the homomorphisms given by its irreducible representations give the bulk reflection coefficients in the presence of elementary boundary conditions. We also show that this algebra is related to traces over intertwiners for mapping class group representations. These results are obtained with the help of manipulations of suitable three-manifolds, making use of the TFT approach and in particular of the results of [30] about factorization. An additional ingredient is the identification of a distinguished subspace $B_{\circ}\left(U_{\imath_{1}}, U_{\bar{\imath}_{1}}, \ldots, U_{\imath_{n}}, U_{\bar{\imath}_{n}}\right)$ of the space $B\left(U_{\imath_{1}}, U_{\bar{\imath}_{1}}, \ldots, U_{\imath_{n}}, U_{\bar{\imath}_{n}}\right)$ of $2 n$-point conformal blocks on the sphere that comes with a natural projection $\pi_{\circ}: B\left(U_{\imath_{1}}, U_{\bar{\imath}_{1}}, \ldots, U_{\imath_{n}}, U_{\bar{\imath}_{n}}\right) \rightarrow B_{\circ}\left(U_{\imath_{1}}, U_{\bar{\imath}_{1}}, \ldots, U_{\imath_{n}}, U_{\bar{\imath}_{n}}\right)$, see the picture (2.14) below. Such a subspace exists because the modular tensor category has a duality.

It is worth pointing out that the TFT approach allows one to compute arbitrary correlation functions of an RCFT. Indeed, together with the chiral symmetry, the structural data of the modular tensor category $\mathcal{C}$ and of the Frobenius algebra $A$ in $\mathcal{C}$ determine the RCFT completely. In particular, the boundary conditions of the CFT are given by the modules over the algebra $A$. The virtue of the classifying algebra is to transfer the problem of doing representation theory for an algebra $A$ in an abstract tensor category to the problem of doing representation theory for an algebra $\mathcal{A}$ over $\mathbb{C}$, keeping at the same time the information about bulk reflection coefficients. Once the structure of $\mathcal{A}$ is known, the boundary conditions of the CFT can be studied without further reference to the category $\mathcal{C}$. In particular, coefficients of boundary states and thus annulus coefficients are encoded in the classifying algebra.

Our general strategy is as follows. We compare boundary and bulk factorization for correlators of bulk fields on a disk. Such correlators are elements of a space of conformal blocks on the sphere. In the TFT approach, they are described by ribbon graphs in a full three-ball $B^{3}$.

We think about this ball $B^{3}$ as a quotient of an interval bundle over the disk, which is obtained in the following manner. The disk is embedded in the equatorial plane of $B^{3}$. Intervals piercing the interior of the disk connect points on the boundary sphere $\partial B^{3}$ of equal longitude and opposite latitude, while the boundary of the disk is connected to the equator of the boundary sphere by little intervals in the equatorial plane. This is illustrated in the pictures (1.1); the first shows the ball $B^{3}$ together with the embedded world sheet (the shaded region) and some of the connecting intervals (intervals intersecting the interior $\mathrm{X} \backslash \partial \mathrm{X}$ of the world sheet shown on the left, and intervals containing a boundary point of $\mathrm{X}$ shown on the right), while the second picture shows the intersection of the ball with a vertical plane containing the north and south poles.
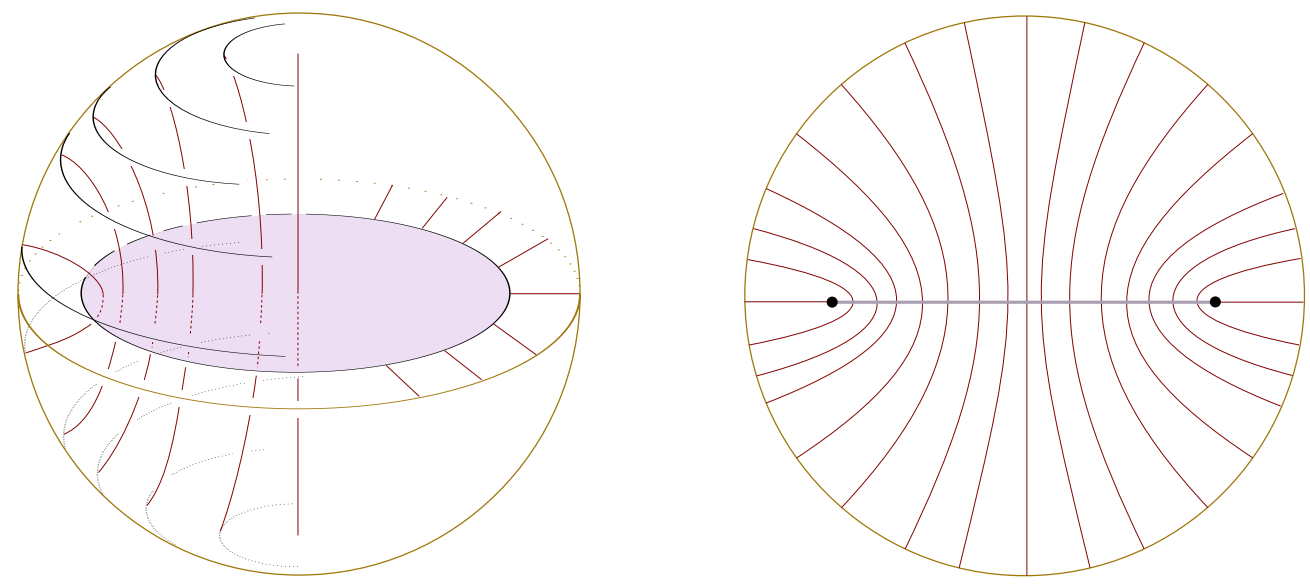

After including, in accordance with the rules (see appendix A.4, or appendix B of [30]) of the 
TFT approach, the appropriate ribbon graph in this three-ball, one takes the invariant of the resulting three-manifold. Thereby the correlator is expressed as an element of the relevant space $B$ of conformal blocks on the two-sphere $S^{2}$. From the correlator one can obtain the corresponding structure constants by gluing to this ball another full ball that contains a standard ribbon graph describing an element of the dual basis in the dual space $B^{*}$. They are thus given as invariants of ribbon graphs in the three-sphere $S^{3}$.

More specifically, we consider a disk with the insertion of an arbitrary number $n$ of bulk fields. Performing $n-1$ boundary factorizations and projecting on the subspace $B_{\circ}\left(U_{\imath_{1}}, \ldots, U_{\imath_{2 n}}\right)$, we find a contribution that is proportional to the product $b_{\imath_{1}} b_{\imath_{2}} \cdots b_{\imath_{n}}$ of $n$ reflection coefficients. Bulk factorization is more subtle. Indeed, the geometry of three-manifolds arising in the TFT approach reveals that such a factorization involves the interchange of a contractible and a non-contractible cycle in a full torus, and thus a modular $S$-transformation. This is sometimes expressed by saying that the $S$-operation interchanges the open and closed channels of the correlator. Cutting out a solid torus from $S^{3}$ and gluing it back with an $S$-transformation yields the closed manifold $S^{2} \times S^{1}$.

The invariant of a ribbon graph in $S^{2} \times S^{1}$ is the trace of an endomorphism of the relevant space of conformal blocks on $S^{2}$. This is the geometric origin of the fact that traces on spaces of conformal blocks on the sphere enter in OPE coefficients. Moreover, after the bulk factorization a single reflection coefficient $b_{k}$ remains as a factor in the expression for the correlator. By extracting its coefficient in the distinguished subspace $B_{\circ}\left(U_{\imath_{1}}, U_{\bar{\imath}_{1}}, \ldots, U_{\imath_{n}}, U_{\bar{\imath}_{n}}\right)$, one arrives at a ribbon graph whose invariant constitutes an intertwiner for the action of the mapping class group on the space $B\left(U_{\imath_{1}}, U_{\imath_{2}}, \ldots, U_{\imath_{n}}, U_{k}\right)$ of conformal $n+1$-point blocks on the sphere. For $n=2$ this intertwiner is the invariant of the following ribbon graph embedded in the three-manifold $S^{2} \times[-1,1]$ :

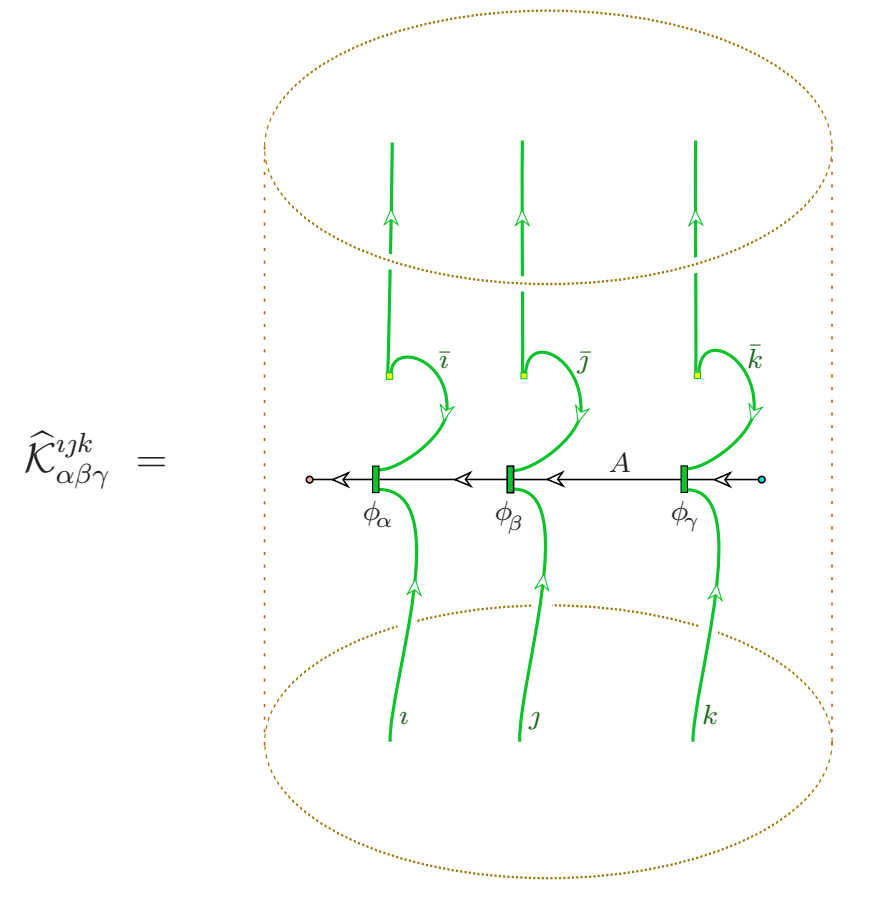

Here the interval $[-1,1]$ is displayed vertically, while the factor $S^{2}$ of the three-manifold corresponds to horizontal planes (for more details see section 3 ). Also, the morphism $\phi_{\alpha}$ is an element of the space of bulk fields with chiral labels $\imath$ and $\bar{\imath}$, and analogously for $\phi_{\beta}$ and $\phi_{\gamma}$; for any choice of these bulk fields one deals with an intertwiner of the mapping class group action on $B\left(U_{i}, U_{j}, U_{k}\right)$. Note that nontriviality of this intertwiner implies reducibility of the mapping class group representation (see [32, 33]). 
Comparing the outcome of boundary and bulk factorization, one arrives in the case $n=2$ at a formula for the structure constants of the classifying algebra. The result is then used to show that the classifying algebra is a semisimple commutative associative algebra over $\mathbb{C}$. This provides precise statements and proofs of the existing conjectures about the classifying algebra and of the relation between boundary states and traces on spaces of conformal blocks. In the Cardy case, in which the torus partition function is given by charge conjugation, the classifying algebra reduces to the Verlinde algebra.

This paper is organized as follows. In sections 2 and 3 we describe the boundary and bulk factorization, respectively, for $n$-point correlators on the disk. Via boundary factorization, these are expressed as a product of $n$ one-point correlators on the disk, see formula (2.18), while via bulk factorization one arrives at an expression involving only a single one-point correlator as a factor, see (3.16). By equating these results we arrive, in section 4, at the classifying algebra $\mathcal{A}$. As a vector space $\mathcal{A}$ consists of those bulk fields which have non-vanishing one-point correlator on the disk. In a basis $\left\{\phi^{\imath, \alpha}\right\}$ of this space the structure constants are given, up to a simple prefactor (see formula (4.1)), by the invariant of the ribbon graph $\mathcal{K}_{\alpha \beta \gamma}^{\imath \jmath k}$ that is obtained by taking the trace over the intertwiner in the ribbon graph (1.2). Taking the trace means that top and bottom of the picture (1.2) are identified so that each of the $i$-, $j$ - and $k$-ribbons forms a loop and one deals with a ribbon graph embedded in the closed three-manifold $S^{2} \times S^{1}$, with $S^{1}$ running vertically. Based on this result for the structure constants we show that the algebra $\mathcal{A}$ is commutative and associative, has a unit, and is semisimple. In particular, the one-dimensional $\mathcal{A}$-representations furnished by the elementary boundary conditions exhaust the (isomorphism classes of) irreducible $\mathcal{A}$-representations.

Throughout this paper methods and results from the TFT approach are used freely. Some pertinent information about this approach is collected in the appendix.

\section{Boundary factorization}

The world sheet of our interest is an oriented disk with boundary condition $M$ and with, for now, an arbitrary number $n$ of bulk field insertions. The boundary condition $M$ is also arbitrary, but kept fixed. After equation (2.15) we will, however, restrict to the case that $M$ is an elementary boundary condition (or in algebraic terms, that $M$ is a simple $A$-module). The goal is to identify quantities - the structure constants of the classifying algebra - in which the dependence on the choice of $M$ has dropped out.

We denote the world sheet by $\mathrm{X} \equiv \mathrm{X}\left(\Phi_{\alpha_{1}}, \Phi_{\alpha_{2}}, \ldots, \Phi_{\alpha_{n}} ; M\right)$, where each of the $n$ field insertions $\Phi_{\alpha_{1}}, \Phi_{\alpha_{2}}, \ldots, \Phi_{\alpha_{n}}$ is a bulk field

$$
\Phi_{\alpha} \equiv\left(U_{\imath}, U_{\jmath}, \phi_{\alpha}\right)
$$

with $U_{\imath}$ and $U_{\jmath}$ simple objects of $\mathcal{C}$ and $\phi_{\alpha}$ an element of the space of $\operatorname{Hom}_{A \mid A}\left(U_{\imath} \otimes^{+} A \otimes^{-} U_{\jmath}, A\right)$ of bimodule morphisms, as described in appendix A.5. The correlator $c\left(\Phi_{\alpha_{1}}, \Phi_{\alpha_{2}}, \ldots, \Phi_{\alpha_{n}} ; M\right)$ for this world sheet can be expressed, according to

$$
c\left(\Phi_{\alpha_{1}}, \Phi_{\alpha_{2}}, \ldots, \Phi_{\alpha_{n}} ; M\right)=Z\left(\mathcal{M}_{\mathrm{X}}\right) 1
$$

as the invariant of a cobordism $\mathcal{M}_{\mathrm{X}}$. The construction of this cobordism, the connecting manifold of $\mathrm{X}$, is summarized in appendix A.4. The connecting manifold is to be regarded as a cobordism from $\emptyset$ to $\partial \mathcal{M}_{\mathrm{X}}$, and $\partial \mathcal{M}_{\mathrm{X}}=\widehat{\mathrm{X}}$ is the double of the world sheet $\mathrm{X}$. Accordingly $Z\left(\mathcal{M}_{\mathrm{X}}\right)$ is a linear map from $\mathbb{C}=Z(\emptyset)$ to the space $Z(\widehat{\mathrm{X}})$ of conformal blocks; in (2.2) this map is applied to the number $1 \in \mathbb{C}$. 
In the case at hand, i.e. for $\mathrm{X}$ a disk, the prescription of appendix A.4 yields for the double $\widehat{X}$ a two-sphere, and for the connecting manifold $\mathcal{M}_{\mathrm{X}}$ a full three-ball. This ball contains a ribbon graph, as shown in the following picture:

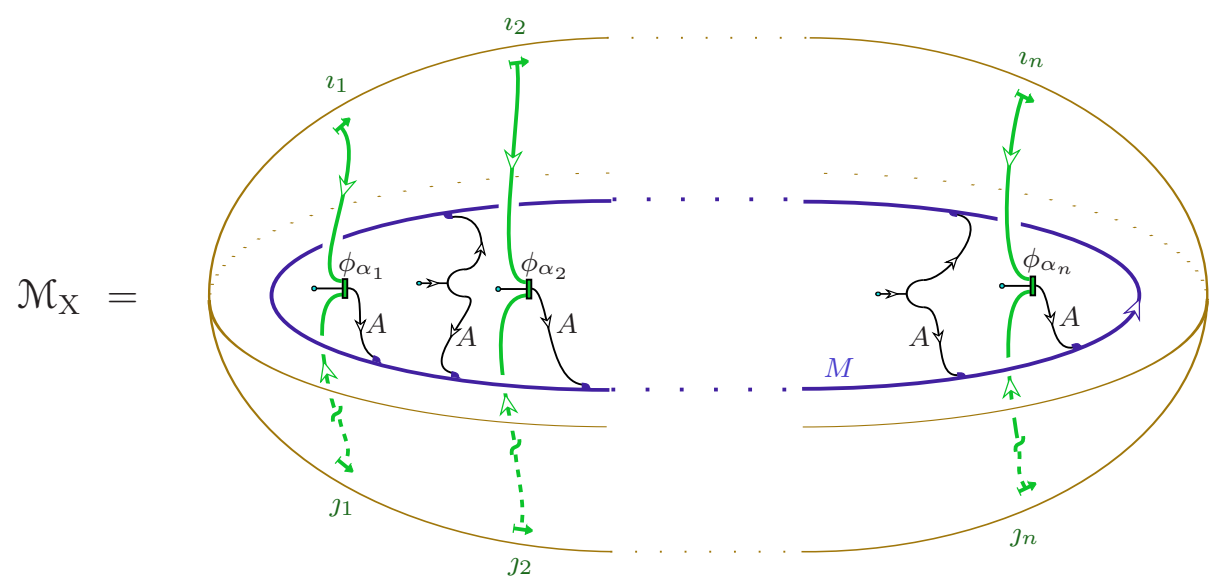

Here the vertical ribbons are labeled by the objects $U_{\imath_{\ell}}$ and $U_{\jmath_{\ell}}$ corresponding to the bulk fields and the annular horizontal ribbon is labeled by the $A$-module $M$, while the other horizontal ribbons, which come from a triangulation of the world sheet, are labeled by the Frobenius algebra $A$ (for more details, compare section 6.3 of [31]).

By examining a cutting of $\mathrm{X}$ along an interval that connects two points on the boundary of the disk, in such a way that the resulting two disks contain $\ell$ and $n-\ell$ bulk insertions, respectively, one obtains the expression

$$
c\left(\Phi_{\alpha_{1}}, \Phi_{\alpha_{2}}, \ldots, \Phi_{\alpha_{n}} ; M\right)=\sum_{q \in \mathcal{I}} \sum_{\gamma, \delta} \operatorname{dim}\left(U_{q}\right)\left(c_{M, M, q}^{\mathrm{bnd}}\right)_{\delta \gamma}^{-1} Z\left(\mathcal{M}_{q \gamma \delta}\right) 1
$$

for the correlator (2.2) (see formulas (2.37), (2.38) and (4.22) of [30]). Here $c^{\text {bnd }}$ is the boundary two-point function (A.12), while for $q$ running over the label set $\mathcal{I}$ for the isomorphism classes of simple objects of $\mathcal{C}$, and for $\gamma$ and $\delta$ running over bases of the morphism spaces $\operatorname{Hom}_{A}\left(M \otimes U_{q}, M\right)$ and $\operatorname{Hom}_{A}\left(M \otimes U_{\bar{q}}, M\right)$, respectively, the cobordism $\mathcal{M}_{q \gamma \delta}$ is given by

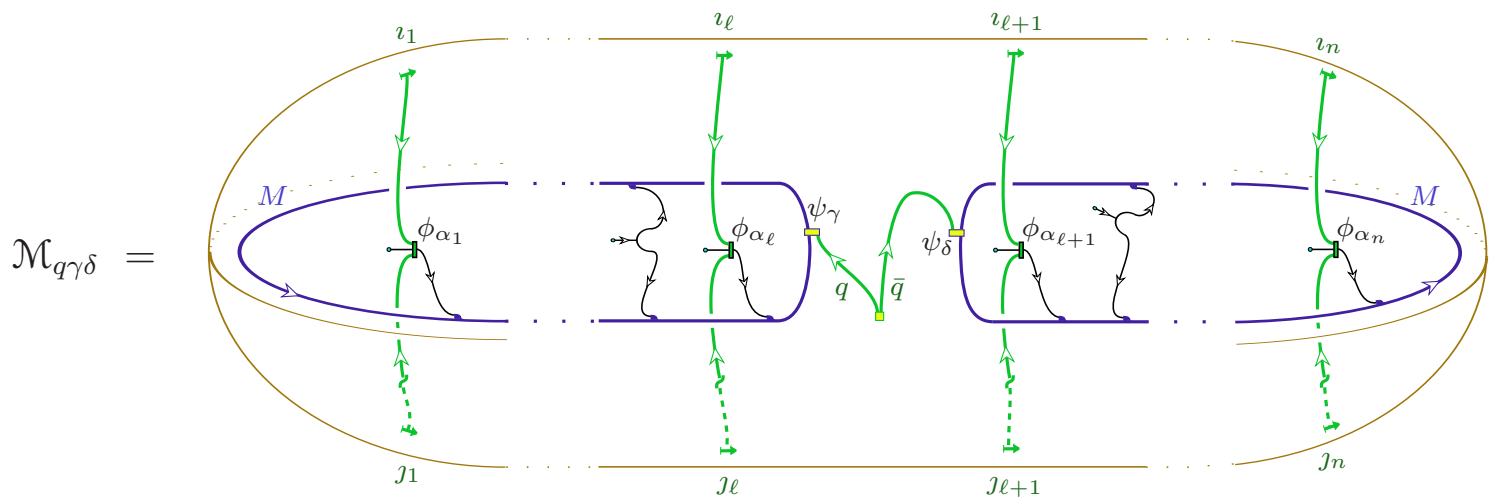

By construction, both $Z\left(\mathcal{M}_{\mathrm{X}}\right) 1$ and $Z\left(\mathcal{M}_{q \gamma \delta}\right) 1$ are elements of the space $B\left(U_{\imath_{1}}, U_{\jmath_{1}}, \ldots, U_{\imath_{n}}, U_{\jmath_{n}}\right)$ of $2 n$-point chiral blocks on the sphere. A standard basis of this space is given by (compare appendix A.1) the vectors $\mathcal{B}\left(\imath_{1}, \jmath_{1}, \ldots, \imath_{n}, \jmath_{n}\right)_{p_{1} p_{2} \ldots p_{2 n-3}, \alpha_{1} \alpha_{2} \ldots \alpha_{2 n-2}}$ which are the invariants of the 
ribbon graphs

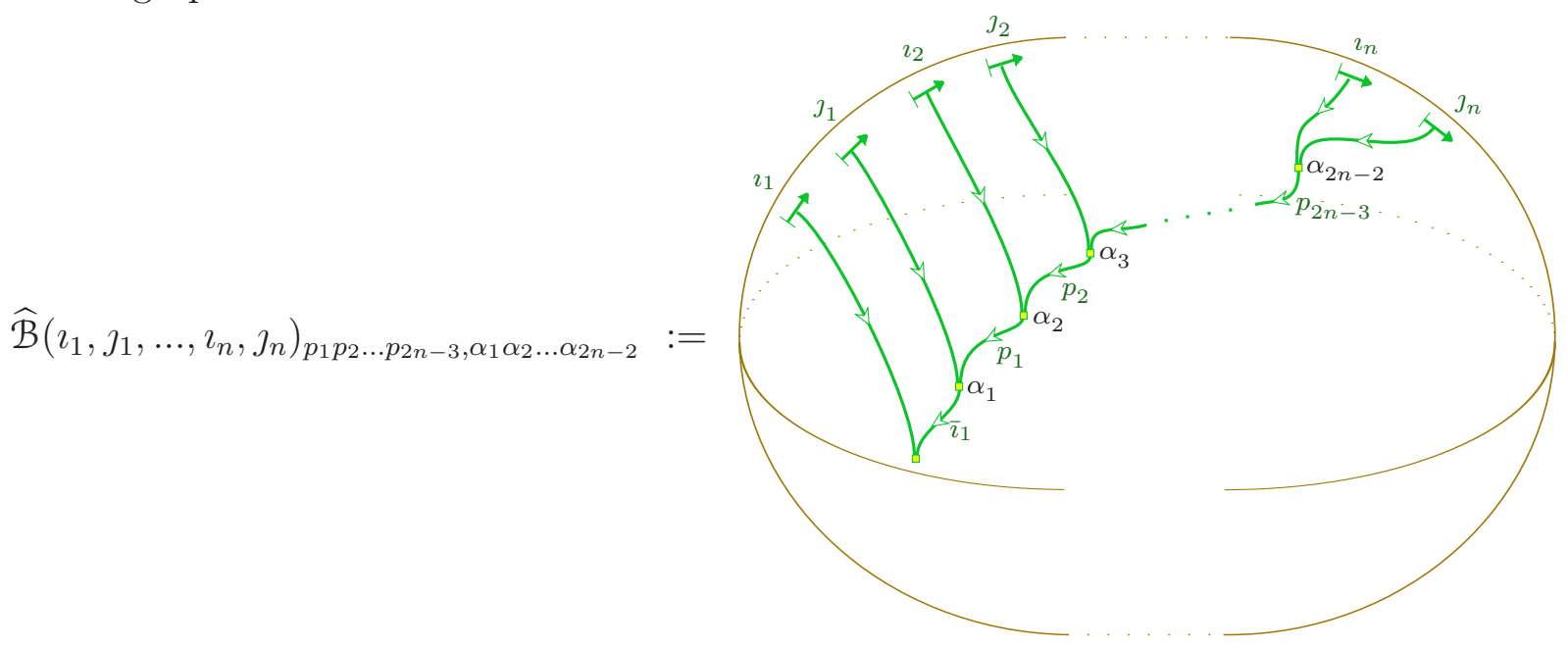

Here $\alpha_{\ell}$ labels a basis of the appropriate morphism space, namely $\operatorname{Hom}\left(U_{p_{2 s}} \otimes U_{\imath_{s+1}}, U_{p_{2 s-1}}\right)$ for $\alpha_{2 s}$, and $\operatorname{Hom}\left(U_{p_{2 s-1}} \otimes U_{\jmath_{s}}, U_{p_{2 s-2}}\right)$ for $\alpha_{2 s-1}$, where $s=1,2, \ldots, n-1$, and where we identify $p_{0} \equiv \bar{\imath}_{1}$ and $p_{2 n-2} \equiv \jmath_{n}$. A vector in $B\left(U_{\imath_{1}}, U_{\jmath_{1}}, \ldots, U_{\imath_{n}}, U_{\jmath_{n}}\right)$ can be expanded with respect to the standard basis (2.6) by evaluating on it an element of the dual basis of the dual space. This amounts to gluing with another three-ball with appropriate ribbon graph and results in a ribbon graph in the closed three-manifold $S^{3}$, the invariant of which gives the coefficient with respect to the chosen basis.

In the following we concentrate on the case $n=2$, the case of arbitrary $n$ being analogous. For $n=2$ we denote the two bulk fields by $\Phi_{\alpha} \equiv\left(\imath, \jmath, \phi_{\alpha}\right)$ and $\Phi_{\beta} \equiv\left(k, l, \phi_{\beta}\right)$, and expand the correlator as

$$
c\left(\Phi_{\alpha}, \Phi_{\beta} ; M\right)=\sum_{p \in \mathcal{I}} \sum_{\varepsilon_{1}, \varepsilon_{2}} c\left(\Phi_{\alpha}, \Phi_{\beta} ; M\right)_{p, \varepsilon_{1} \varepsilon_{2}} Z\left(\mathcal{B}(\jmath l l k)_{p, \varepsilon_{1} \varepsilon_{2}}\right)
$$

where $\mathcal{B}(\jmath l k)_{p, \varepsilon_{1} \varepsilon_{2}}$ are basis elements of the form (2.6) for the case of four-point blocks. By evaluating an element of the dual basis on both sides of this equality, one obtains the expression

$$
c\left(\Phi_{\alpha}, \Phi_{\beta} ; M\right)_{p, \varepsilon_{1} \varepsilon_{2}}=\sum_{q \in \mathcal{I}} \sum_{\gamma, \delta} \operatorname{dim}\left(U_{q}\right)\left(c_{M, M, q}^{\mathrm{bnd}}\right)_{\delta \gamma}^{-1} Z\left(\mathcal{M}_{p, \varepsilon_{1} \varepsilon_{2}}^{q, \gamma \delta}\right)
$$

for the coefficients in (2.7), with $\mathcal{M}_{p, \varepsilon_{1} \varepsilon_{2}}^{q, \gamma \delta}$ the following ribbon graph in $S^{3}$ :

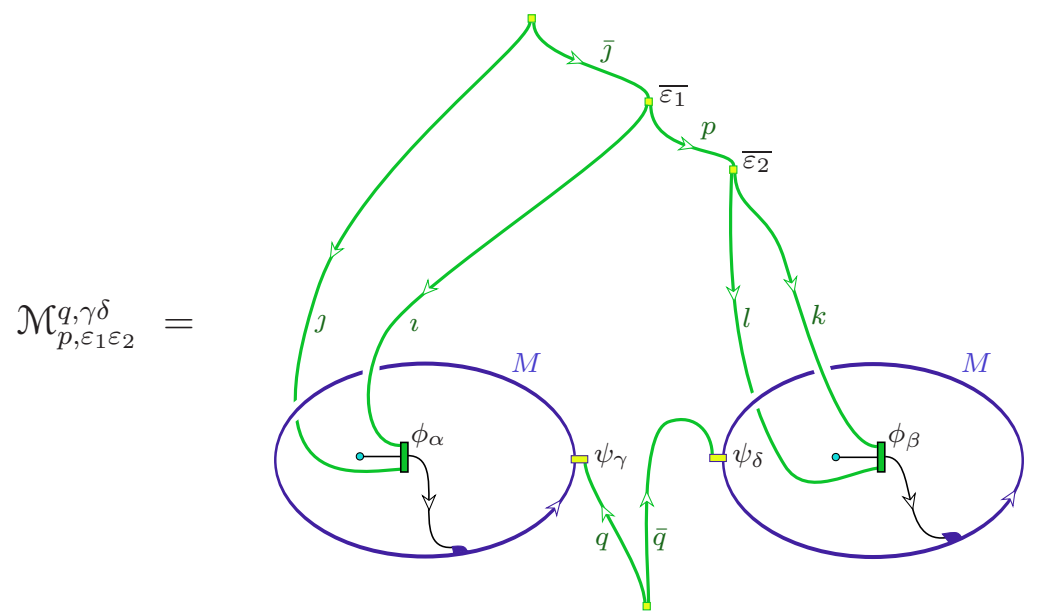


Here the morphisms $\psi_{\gamma}$ and $\psi_{\delta}$ correspond to boundary fields as described in appendix A.5. The invariant of the graph (2.9) can be computed by projecting it in a non-singular manner to a plane and interpreting the resulting planar picture as a morphism in the category $\mathcal{C}$. With a suitable choice of projection the resulting morphism in $\operatorname{Hom}(\mathbf{1}, \mathbf{1})$ looks as follows:

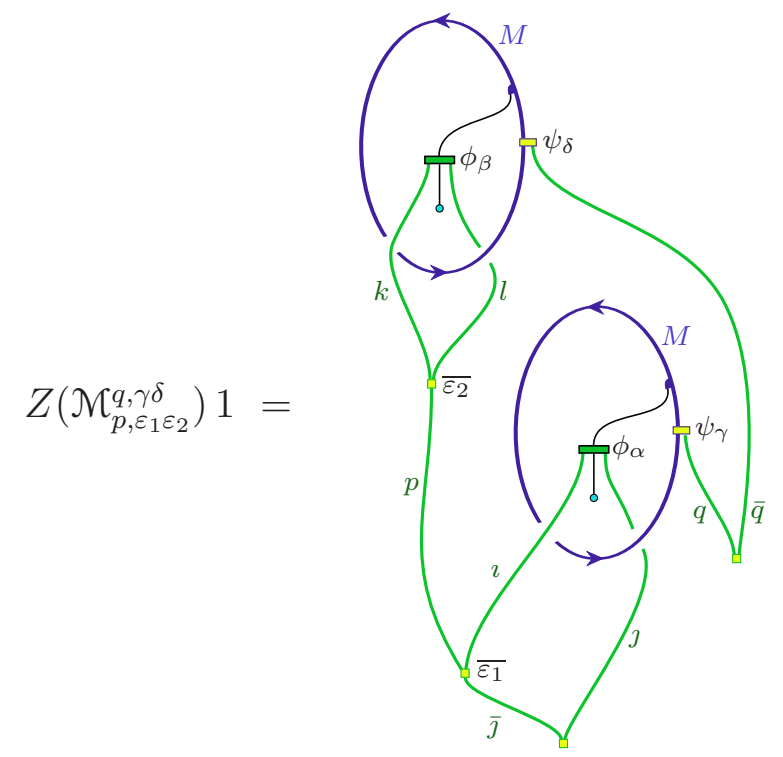

Using dominance in the space $\operatorname{Hom}\left(U_{p} \otimes U_{\bar{q}}, U_{p} \otimes U_{\bar{q}}\right)$ one sees that only the term with $q=p$ contributes in the $q$-summation in (2.7). Further, for this term one obtains

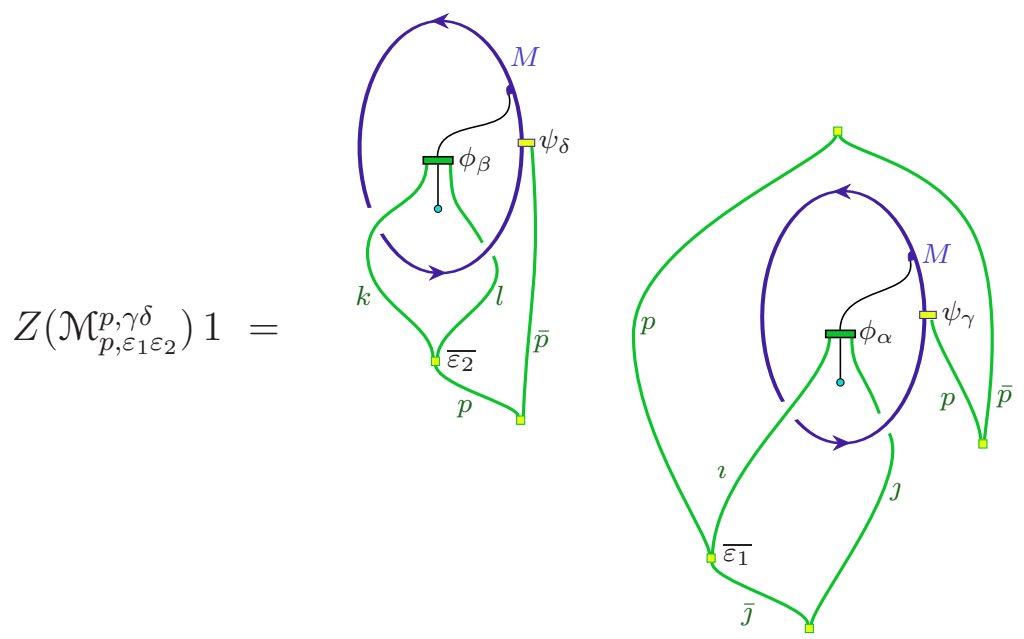

Next we note that the summation over the label $p$ in the expression (2.7) for the correlator corresponds to the decomposition

$$
\operatorname{Hom}\left(\mathbf{1}, U_{\imath} \otimes U_{\jmath} \otimes U_{k} \otimes U_{l}\right) \cong \bigoplus_{p \in \mathcal{I}} \operatorname{Hom}\left(U_{p}^{\vee}, U_{\imath} \otimes U_{\jmath}\right) \otimes_{\mathbb{C}} \operatorname{Hom}\left(U_{p}, U_{k} \otimes U_{l}\right)
$$

(which is equivalent to (A.4) for $m=2$ ). The information we are mainly interested in is supplied by the contribution of the vacuum channel $p=0$ to the correlator (2.7), corresponding to the summand $p=0$ in the decomposition (2.12). For any even $m=2 n$ this contribution can be extracted unambiguously because the space $B\left(U_{\imath_{1}}, U_{\jmath_{1}}, \ldots, U_{\imath_{n}}, U_{\jmath_{n}}\right)$ of conformal blocks has a distinguished 
subspace $B_{\circ}\left(U_{\imath_{1}}, U_{\jmath_{1}}, \ldots, U_{\imath_{n}}, U_{\jmath_{n}}\right)$ that corresponds to the vacuum channel; it is obtained by choosing in (2.6) all $p_{s}$ with odd label $s$ to correspond to the identity field, i.e. by taking $p_{2 s-1}=0$ for all $s=1,2, \ldots, n-1$. The subspace $B_{\circ}\left(U_{\imath_{1}}, U_{\jmath_{1}}, \ldots, U_{\imath_{n}}, U_{\jmath_{n}}\right)$ is zero unless $\jmath_{k}=\bar{\imath}_{k}$ for all $k=1,2, \ldots, n$, and in the latter case $B_{\circ}\left(U_{\imath_{1}}, U_{\jmath_{1}}, \ldots, U_{\imath_{n}}, U_{\jmath_{n}}\right)=B_{\circ}\left(U_{\imath_{1}}, U_{\bar{\imath}_{1}}, \ldots, U_{\imath_{n}}, U_{\bar{\imath}_{n}}\right)$ is one-dimensional, the single contribution coming from $p_{2 k}=\bar{\imath}_{k}$ for all $k=1,2, \ldots, n-2$ (as well as with all multiplicity labels having only a single possible value, which we indicate by the symbol 'o'). We denote by

$$
\mathcal{B}\left(\imath_{1} \bar{l}_{1} \imath_{2} \bar{\imath}_{2} \ldots \imath_{n} \bar{\imath}_{n}\right)_{0 \bar{\imath}_{2} 0 \bar{\imath}_{3} \ldots 0 \bar{\imath}_{n-1} 0, \circ \ldots \circ \circ}=: \mathcal{B}\left(\imath_{1} \bar{\imath}_{1} \imath_{2} \bar{\imath}_{2} \ldots \imath_{n} \bar{\imath}_{n}\right)_{0}
$$

the corresponding basis element of the space $B\left(U_{\imath_{1}}, \ldots, U_{\bar{\imath}_{n}}\right)$. In terms of the pictorial description (2.6) of the basis of $B\left(U_{\imath_{1}}, U_{\jmath_{1}}, \ldots, U_{\imath_{n}}, U_{\jmath_{n}}\right)$, the basis elements (2.13) are given by the invariants of the ribbon graphs

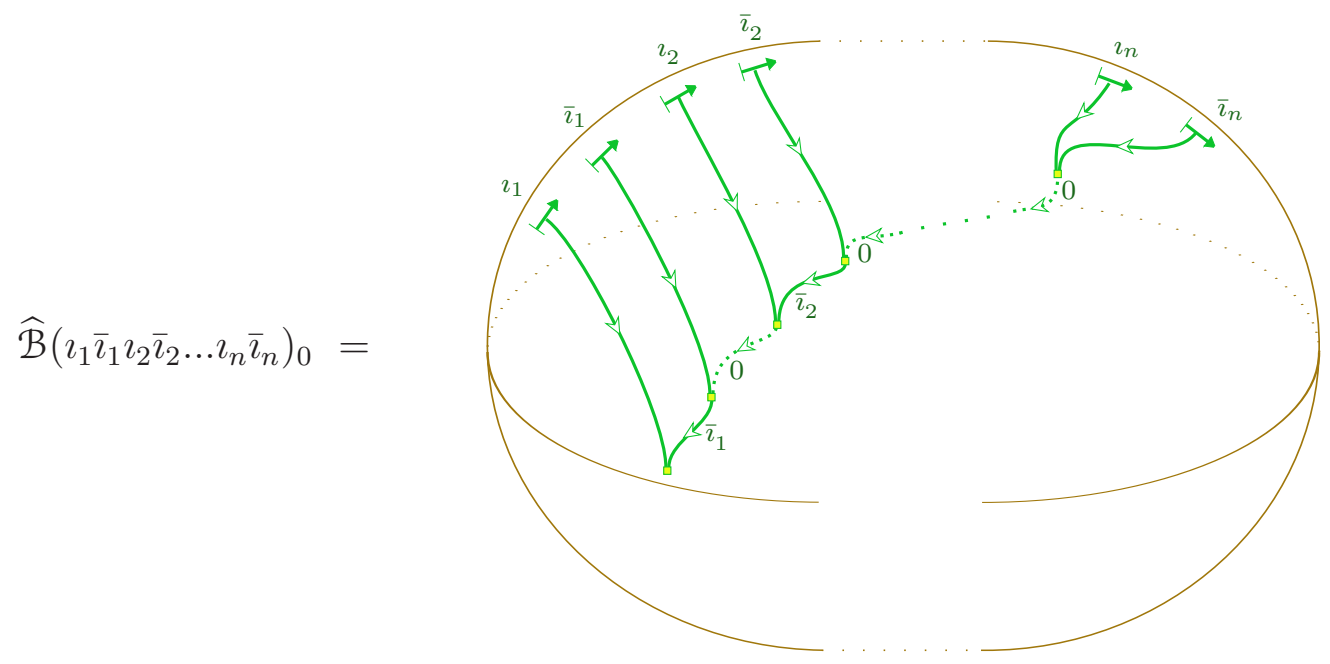

The subspace $B_{\circ}\left(U_{\imath_{1}}, U_{\jmath_{1}}, \ldots, U_{\imath_{n}}, U_{\jmath_{n}}\right)$ exists as a consequence of the fact that the category $\mathcal{C}$ has a duality. It comes with a canonical projection $\pi_{\circ}$ from $B\left(U_{\imath_{1}}, U_{\jmath_{1}}, \ldots, U_{\imath_{n}}, U_{\jmath_{n}}\right)$ such that $\pi_{\circ} \circ e=\mathrm{id}_{B_{\circ}\left(U_{\imath_{1}}, U_{\jmath_{1}}, \ldots, U_{\imath_{n}}, U_{J_{n}}\right)}$ for $e: B_{\circ}\left(U_{\imath_{1}}, U_{\jmath_{1}}, \ldots, U_{\imath_{n}}, U_{\jmath_{n}}\right) \hookrightarrow B\left(U_{\imath_{1}}, U_{\jmath_{1}}, \ldots, U_{\imath_{n}}, U_{\jmath_{n}}\right)$. Indeed, every basis of $B\left(U_{\imath_{1}}, U_{\jmath_{1}}, \ldots, U_{\imath_{n}}, U_{j_{n}}\right)$ that consists of tensor products of basis elements of three-point blocks contains the element (2.13), and in the basis transformation between any two such bases this element is mapped to itself and does not appear at all in the transformation of any other basis element.

Next we observe, by comparison with formula (4.23) of [31], that the two factors on the right hand side of (2.11) are (apart from some additional fusing moves of the type displayed in (A.3) which are implemented easily) just the structure constants for correlators of a disk with one bulk field and one boundary field insertion. For the first factor, the fields are $\Phi_{\beta}$ and $\Psi_{\delta}$, while for the second they are $\Phi_{\alpha}$ and $\Psi_{\gamma}$. Putting the various ingredients of the coefficients (2.8) together, we then arrive at the formula

$$
c\left(\Phi_{\alpha}, \Phi_{\beta} ; M\right)_{0} \equiv \pi_{\circ}\left(c\left(\Phi_{\alpha}, \Phi_{\beta} ; M\right)\right)=\sum_{\gamma, \delta}\left(c_{M, M, 0}^{\mathrm{bnd}}\right)_{\delta \gamma}^{-1} c\left(\Phi_{\alpha}, \Psi_{\gamma} ; M\right) c\left(\Phi_{\beta}, \Psi_{\delta} ; M\right)
$$

for the coefficients with $p=0$.

Let us now restrict our attention to the case of a simple $A$-module $M$. In this case the space $\operatorname{Hom}_{A}\left(M \otimes U_{0}, M\right)$ in which the morphism $\psi_{\gamma}$ takes values is one-dimensional, and it has the unit constraint of the category $\mathcal{C}$ as a canonical basis. As a consequence, for simple $M$ the two disk correlators on the right hand side of (2.15) can be canonically described by complex numbers 
$c\left(\Phi_{\alpha} ; M\right)$ and $c\left(\Phi_{\beta} ; M\right)$, and the two-point function of boundary fields canonically reduces to a complex number as well, see (A.13). Thus for simple $M$ we arrive at the expression

$$
c\left(\Phi_{\alpha}, \Phi_{\beta} ; M\right)_{0}=\left(c_{M, 0}^{\mathrm{bnd}}\right)^{-1} c\left(\Phi_{\alpha} ; M\right) c\left(\Phi_{\beta} ; M\right) .
$$

The generalization of this result to an arbitrary number $n$ of bulk fields on the disk is direct. The coefficient $c\left(\Phi_{\alpha_{1}}, \Phi_{\alpha_{2}}, \ldots, \Phi_{\alpha_{n}} ; M\right)_{0}$ corresponding to the one-dimensional vacuum-channel subspace with basis element $\mathcal{B}\left(\bar{\imath}_{1} \imath_{1} \bar{\imath}_{2} \imath_{2} \ldots \bar{\nu}_{n} \imath_{n}\right)_{0 \imath_{2} 0 \ldots \imath_{n-1} 0}$ is

$$
c\left(\Phi_{\alpha_{1}}, \Phi_{\alpha_{2}}, \ldots, \Phi_{\alpha_{n}} ; M\right)_{0}=\left(\left(c_{M, 0}^{\mathrm{bnd}}\right)^{-1}\right)^{n-1} \prod_{i=1}^{n} c\left(\Phi_{\alpha_{i}} ; M\right) .
$$

In short, the vacuum-channel coefficient of an $n$-point correlator of bulk fields on the disk is the product over the boundary state coefficients $c\left(\Phi_{\alpha_{i}} ; M\right)$ for each of the field insertions, up to a normalization factor given by a power of the inverse of the boundary vacuum two-point function.

Now observe that the latter prefactor can actually be absorbed into a simple change of normalization, namely by dividing the correlators of bulk fields on a disk by the boundary vacuum two-point function $c_{M, 0}^{\text {bnd }}$ :

$$
b\left(\Phi_{\alpha_{1}}, \Phi_{\alpha_{2}}, \ldots, \Phi_{\alpha_{n}} ; M\right)_{0}=\prod_{i=1}^{n} b\left(\Phi_{\alpha_{i}} ; M\right)
$$

with $b\left(\Phi_{\alpha_{1}}, \ldots, \Phi_{\alpha_{n}} ; M\right)_{0}=c\left(\Phi_{\alpha_{1}}, \ldots, \Phi_{\alpha_{n}} ; M\right)_{0} / c_{M, 0}^{\text {bnd }}$. Moreover, the re-normalized boundary state coefficients

$$
b_{M}^{\imath, \alpha} \equiv b\left(\Phi_{\alpha} ; M\right)=\left(c_{M, 0}^{\mathrm{bnd}}\right)^{-1} c\left(\Phi_{\alpha}^{\imath \bar{p}} ; M\right)
$$

are nothing but the reflection coefficients which appear (see [34] or section 2.7 of [35]) in the expansion into boundary fields of the bulk field $\Phi_{\alpha}$ when it comes close to the boundary.

\section{Bulk factorization}

Our next goal is to derive another equality for the same disk correlator (2.17) for $n$ bulk fields, this time making use of bulk factorization. One expects that the operator products of bulk fields enter in the resulting formulas. In fact, one of the main results of this paper are explicit expressions for such a factorization. The result has a remarkably simple structure, involving traces of intertwiners for an action of the mapping class group of a sphere with marked points.

Given any world sheet $\mathrm{X}$, the examination of a cutting of $\mathrm{X}$ along a circle $S$ in the interior of $\mathrm{X}$ leads to the expression

$$
c(\mathrm{X})=\sum_{q_{1}, q_{2} \in \mathcal{I}} \sum_{\gamma, \delta} \operatorname{dim}\left(U_{q_{1}}\right) \operatorname{dim}\left(U_{q_{2}}\right)\left(c_{q_{1}, q_{2}}^{\mathrm{bulk}}\right)_{\delta \gamma}^{-1} Z\left(\mathcal{M}_{\mathrm{X} ; q_{1} q_{2} \gamma \delta}\right) 1
$$

for the correlator of X. Here $c^{\text {bulk }}$ is the bulk two-point function described in (A.15), while the labels $\gamma$ and $\delta$ run over bases of the bimodule morphism spaces $\operatorname{Hom}_{A \mid A}\left(U_{q_{1}} \otimes^{+} A \otimes^{-} U_{q_{2}}, A\right)$ and $\operatorname{Hom}_{A \mid A}\left(U_{\bar{q}_{1}} \otimes^{+} A \otimes^{-} U_{\bar{q}_{2}}, A\right)$, respectively. For any $q_{1}, q_{2} \in \mathcal{I}$ and any value of $\gamma$ and $\delta$ the manifold

$\mathcal{M}_{\mathrm{X} ; q_{1} q_{2} \gamma \delta}$ is a cobordism from $\emptyset$ to $\partial \mathcal{M}_{\mathrm{X} ; q_{1} q_{2} \gamma \delta}=\widehat{\mathrm{X}}$, which is just what is needed for $Z\left(\mathcal{M}_{\mathrm{X} ; q_{1} q_{2} \gamma \delta}\right) 1$ to be an element of the same space of conformal blocks as the correlator $c(\mathrm{X})$. 
Implementing the construction summarized in appendix A.4, one obtains the following prescription for obtaining the cobordism $\mathcal{M}_{\mathrm{X} ; q_{1} q_{2} \gamma \delta}$ :

- First, remove from the connecting manifold $\mathcal{M}_{\mathrm{X}}$ for $\mathrm{X}$ the union $\mathrm{Y}_{S}$ of all connecting intervals that intersect the image of the cutting circle $S \subset \mathrm{X}$ under the embedding $\mathrm{I}: \mathrm{X} \rightarrow \mathcal{M}_{\mathrm{X}}$. More explicitly, $\mathrm{Y}_{S}$ is the preimage

$$
\mathrm{Y}_{S}:=\pi_{\mathrm{X}}^{-1}(\mathrm{I}(S)) \subset \mathcal{M}_{\mathrm{X}}
$$

of $\mathrm{I}(S)$ under the canonical projection $\pi_{\mathrm{X}}$ from $\mathcal{M}_{\mathrm{X}}$ to $\mathrm{I}(\mathrm{X})$; by construction, $\mathrm{Y}_{S}$ is an annulus, and $\partial \mathrm{Y}_{S} \subset \partial \mathcal{M}_{\mathrm{X}}$.

By a judicious choice of the triangulation $\Gamma$ of $\mathrm{X}$, without loss of generality we can assume that the circle $\mathrm{I}(S)$ intersects precisely one of the $A$-ribbons that belong to the triangulation, i.e. $\mathrm{I}(S) \cap \Gamma$ consists of a single arc, which we denote by $\ell_{S}$.

As an illustration, when $\mathrm{X}$ is a disk, the annulus $\mathrm{Y}_{S}$ and $\operatorname{arc} \ell_{S}$ for one possible choice of $S$ and $\Gamma$ look as follows:

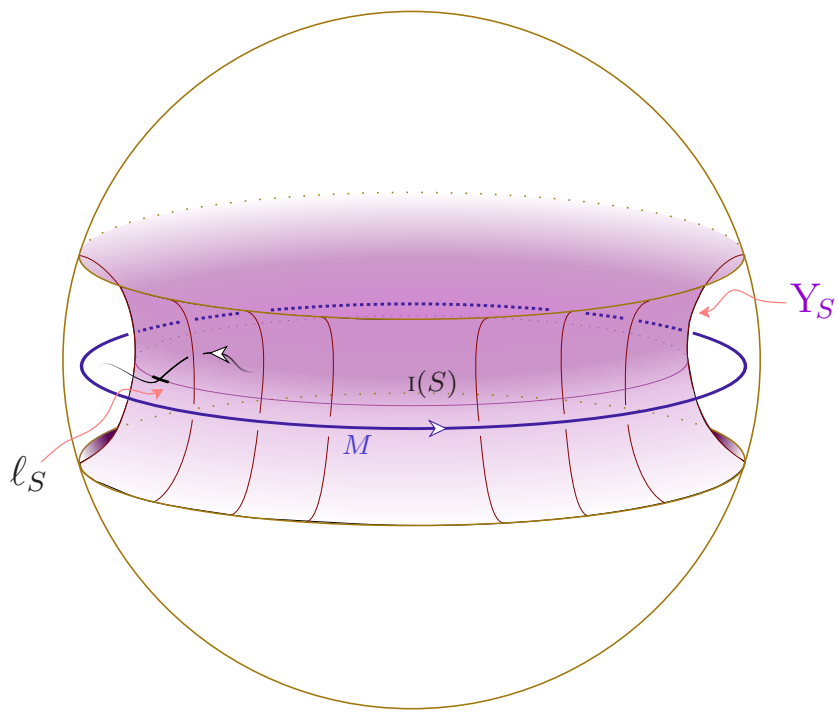

- Take the closure of the so obtained manifold. This results in a three-manifold (with corners) $\mathcal{M}_{\mathrm{X}}^{\circ}$ whose boundary contains two disjoint copies $\mathrm{Y}_{S}^{1,2}$ of $\mathrm{Y}_{S}$. (If $\mathcal{M}_{\mathrm{X}}^{\circ}$ is disconnected, then $\mathrm{Y}_{S}^{1}$ and $\mathrm{Y}_{S}^{2}$ lie in two different connected components.) Here the labeling is chosen in such a way that of the two copies $\ell_{S}^{1,2} \subset Y_{S}^{1,2}$ of $\ell_{S}$, the one denoted by $\ell_{S}^{1}$ belongs to an $A$-ribbon whose core is oriented outwards, while the one denoted by $\ell_{S}^{2}$ belongs to an $A$-ribbon with inwards-oriented core.

- For $\kappa \in\{1,2\}$, identify the surfaces $\mathrm{Y}_{S}^{\kappa} \subset \partial \mathcal{N}_{\mathrm{X}}^{\circ}$ with the distinguished annular part $\mathrm{Y}_{\mathcal{T}}^{\kappa}$ of the boundary of another three-manifold (with corners) $\mathcal{T}_{q_{1} q_{2} \gamma \delta}$. This manifold $\mathcal{T}_{q_{1} q_{2} \gamma \delta}$ is a full torus (with corners) containing a specific ribbon graph that includes in particular the morphisms $\phi_{\gamma}$ and $\phi_{\delta}$ for two additional bulk field insertions. Describing the full torus as a cylinder with top 
and bottom identified, $\mathcal{T}_{q_{1} q_{2} \gamma \delta}$ looks as follows:

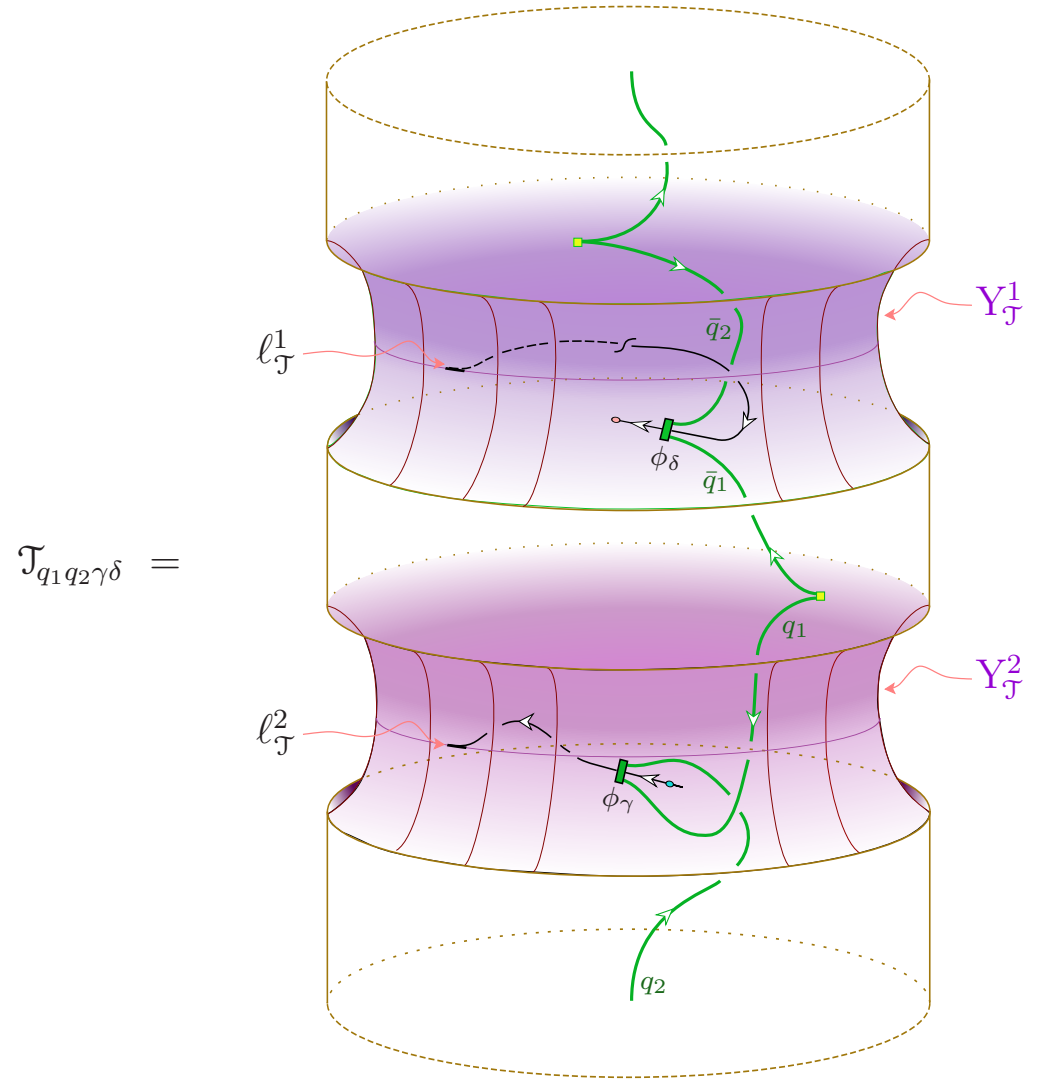

(Note that due to the identification of top and bottom the $q_{1^{-}}$and $q_{2}$-ribbons form a loop.) The identification of $\mathrm{Y}_{S}^{\kappa}$ with $\mathrm{Y}_{\mathcal{T}}^{\kappa}$ is performed in such a manner that, for $\kappa=1,2$, the copy $\ell_{S}^{\kappa} \subset \mathrm{Y}_{S}^{\kappa}$ of $\ell_{S}$ gets identified with the arc $\ell_{\mathcal{T}}^{\kappa}$ on the boundary of $\mathcal{T}_{q_{1} q_{2} \gamma \delta}$ at which an $A$-ribbon ends, and also such that the pieces of $A$-ribbon that get connected this way have matching 2 -orientations.

- Finally, $\mathcal{M}_{\mathrm{X} ; q_{1} q_{2} \gamma \delta}$ is the resulting three-manifold, regarded as a cobordism from the empty set to the two-manifold $\partial \mathcal{M}_{\mathrm{X} ; q_{1} q_{2} \gamma \delta}=\partial \mathcal{M}_{\mathrm{X}}$ which is homeomorphic to the double $\widehat{\mathrm{X}}$ of the world sheet.

This prescription for $\mathcal{M}_{\mathrm{X} ; q_{1} q_{2} \gamma \delta}$ can be understood as the result of composing two cobordisms: First, the correlator $c\left(\mathrm{X}_{q_{1} q_{2} \gamma \delta}\right)$ for a world sheet $\mathrm{X}_{q_{1} q_{2} \gamma \delta}$ which is obtained by a cutting of $\mathrm{X}$ and which as a result of the cutting procedure contains two additional bulk field insertions $\Phi_{\gamma}$ and $\Phi_{\delta}$. And second, the gluing homomorphism, which is the invariant of a cobordism from the double $\widehat{\mathrm{X}}_{q_{1} q_{2} \gamma \delta}$ of the cut world sheet to the double $\widehat{\mathrm{X}}$ of the original world sheet. Thus in particular it follows by construction that indeed $\partial \mathcal{M}_{\mathrm{X} ; q_{1} q_{2} \gamma \delta} \cong \widehat{\mathrm{X}}$. It must however be pointed out that the prescription above constitutes a shortcut that only describes the final result of the manipulations which are involved in the factorization. For instance, in order to understand the origin of the three-manifold $\mathcal{T}_{q_{1} q_{2} \gamma \delta}$, which is the part of $\mathcal{M}_{\mathrm{X} ; q_{1} q_{2} \gamma \delta}$ that in [30] is displayed as the picture (5.9), it is essential that the actual cutting of $\mathrm{X}$ is not performed along a circle, but rather involves an annular collar around that circle, as illustrated in picture (5.2) of [30]. For a few more details see appendix A.4. while for a full derivation, the reader is invited to consult section 5 of [30].

Compatibility with factorization is an extremely tight constraint on correlators, as it relates correlators on world sheets with different topology. What renders the proof of factorization possible is the fundamental insight implicit in the proof in [30] that factorization is a local issue on the world sheet - the required manipulations of three-manifolds only involve the fibers of $\mathcal{M}_{\mathrm{X}}$ over 
some suitable neighbourhood of the cutting circle $S$. For our present purposes, however, we want to compare the actual values of correlators on $\mathrm{X}$, which is a global issue on $\mathrm{X}$. As a consequence, the whole three-manifold $\mathcal{M}_{\mathrm{X} ; q_{1} q_{2} \gamma \delta}$, and accordingly also the whole three-manifold corresponding to the gluing homomorphism, enter our analysis.

Let us now apply the general prescription given above to the situation of our interest, i.e. to the correlator of $n$ bulk fields on the disk, see (2.2). The cutting circle $S$ relevant for the bulk factorization runs parallel to the boundary ribbon, as indicated in

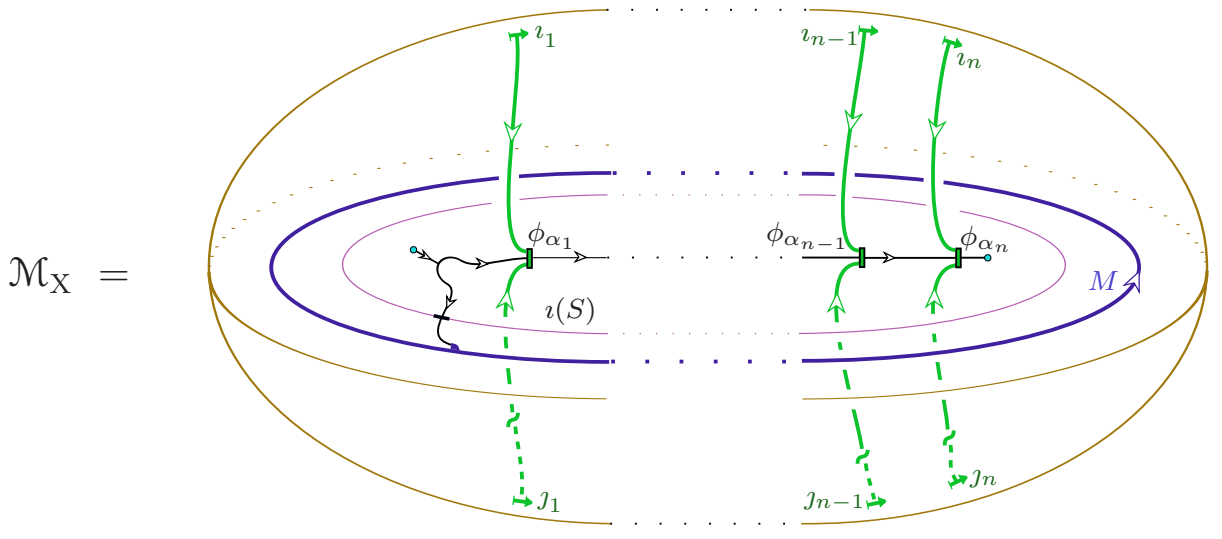

The corresponding annulus $\mathrm{Y}_{S} \subset \mathcal{M}_{\mathrm{X}}$ then looks as in figure (3.3). It follows that the three-manifold $\mathcal{M}_{\mathrm{X}}^{\circ}$, whose boundary contains two disjoint copies $\mathrm{Y}_{S}^{1,2}$ of $\mathrm{Y}_{S}$, is disconnected. Its two connected components are given by a 'nibbled apple'

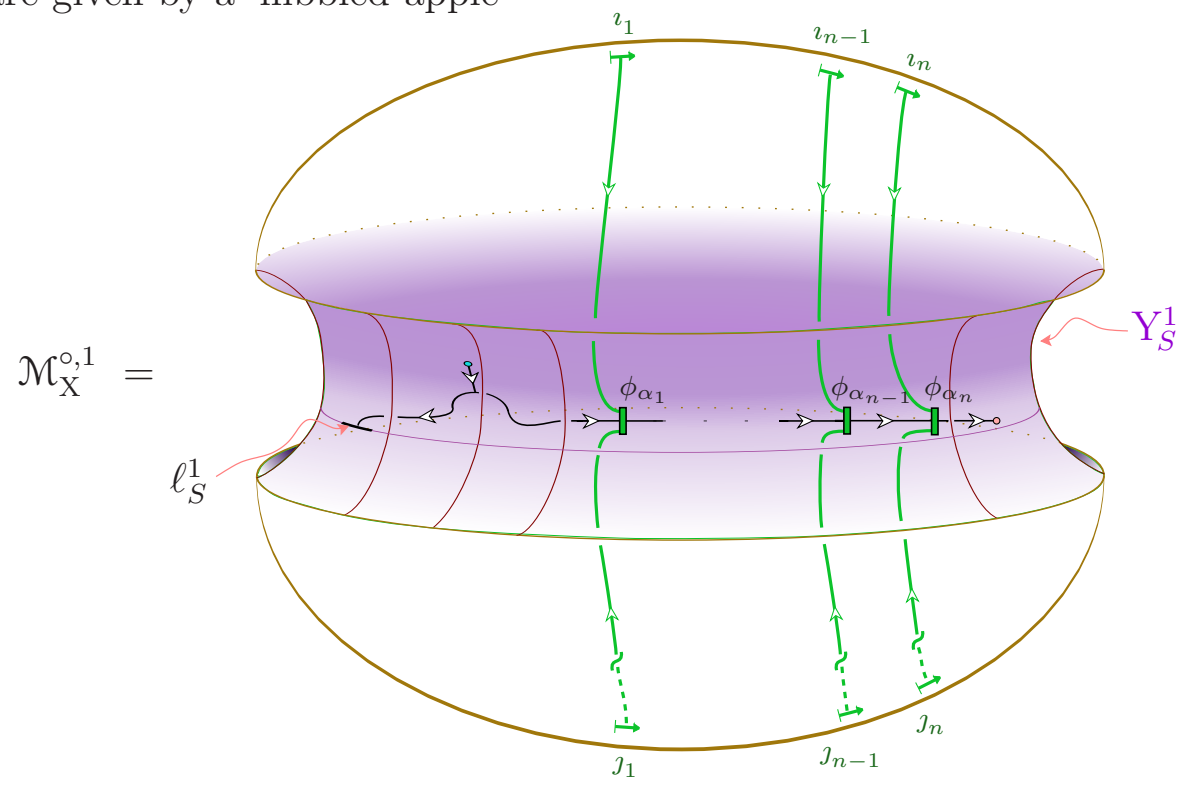

and a 'bigonal doughnut'

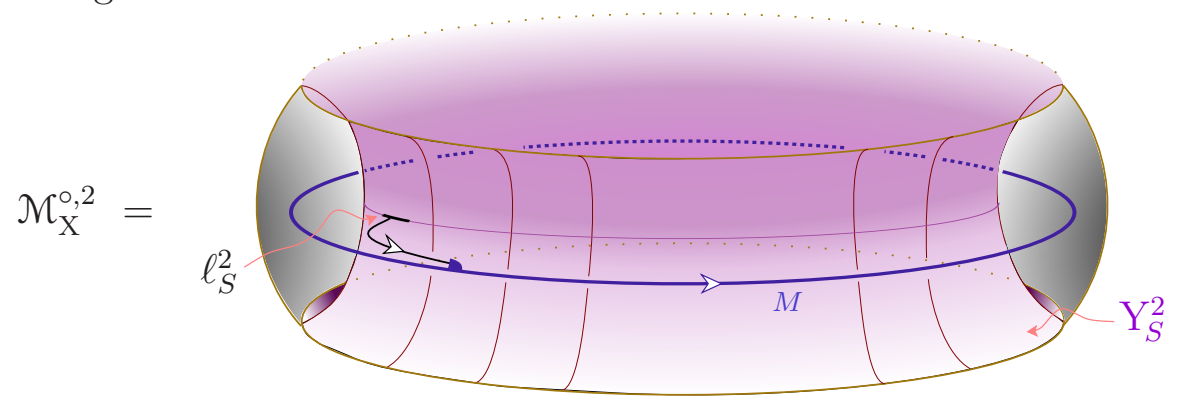


Here we indicate in particular the two copies $\mathrm{Y}_{S}^{1}$ and $\mathrm{Y}_{S}^{2}$ of $\mathrm{Y}_{S}$ (they are shaded) as well as the $\operatorname{arcs} \ell_{S}^{1,2}$ contained in them. For completeness, let us also display schematically the intersection of $\mathcal{M}_{\mathrm{X}}^{\circ, 1}$ and $\mathcal{M}_{\mathrm{X}}^{\circ, 2}$ with a vertical plane containing the north and south poles of the three-ball:

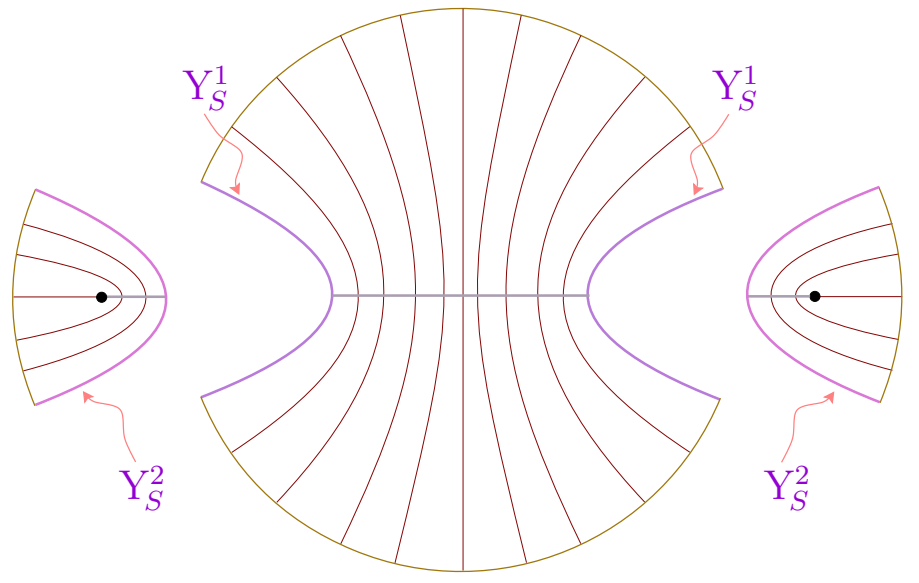

The manifold $\mathcal{M}_{\mathrm{X} ; q_{1} q_{2} \gamma \delta}$ is now obtained by identifying the annuli $\mathrm{Y}_{S}^{1}$ and $\mathrm{Y}_{S}^{2}$ with the corresponding parts $\mathrm{Y}_{\mathcal{T}}^{1}$ and $\mathrm{Y}_{\mathcal{T}}^{2}$ of the manifold $\mathcal{T}_{q_{1} q_{2} \gamma \delta}$ given in (3.4). Implementing this identification is easy in the case of the boundary $\mathrm{Y}_{S}^{2}$ of the bigonal doughnut (3.7); it results in the manifold $\mathcal{N}_{\mathrm{X} ; q_{1} q_{2} \gamma \delta}$ that is shown in the following picture (where top and bottom are still identified):

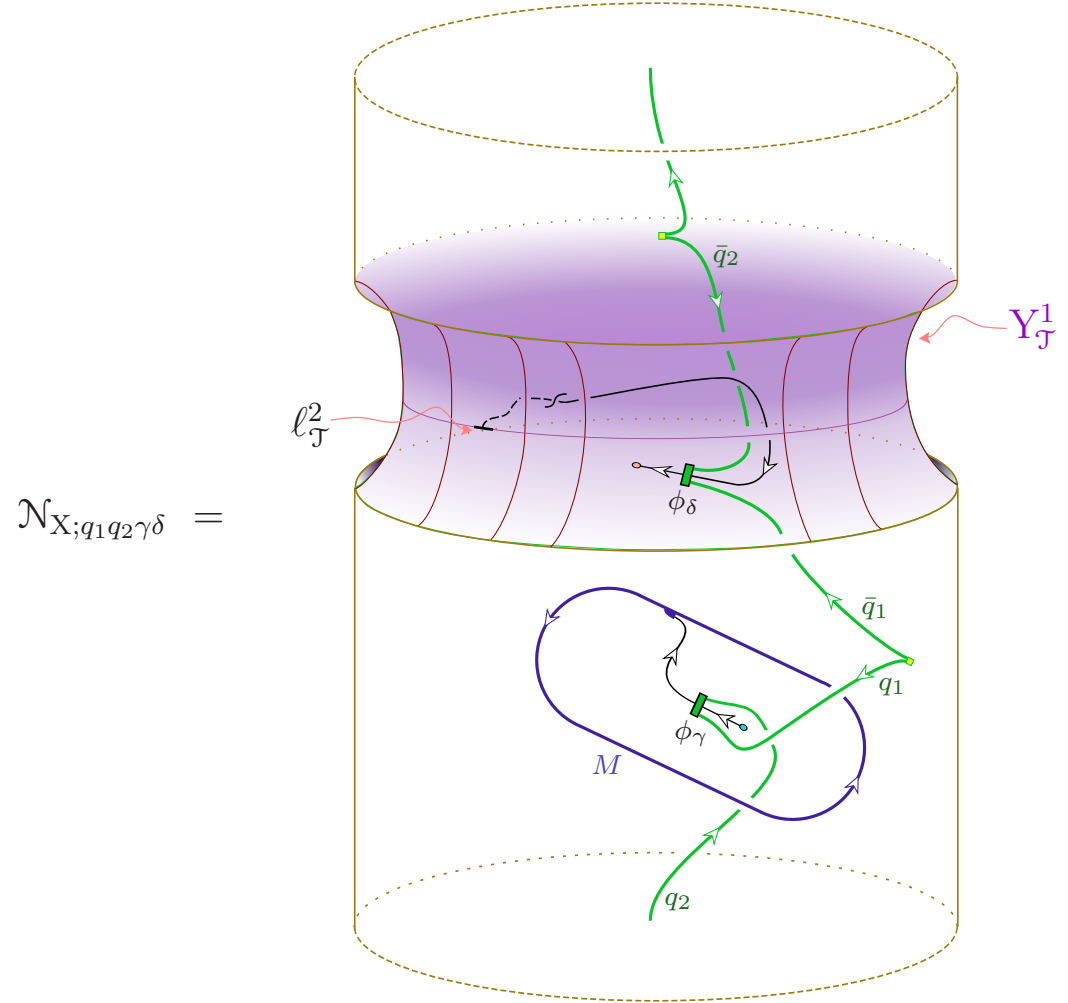

Identifying the boundary $\mathrm{Y}_{S}^{1}$ of the nibbled apple (3.6) with $\mathrm{Y}_{\mathcal{T}}^{1}$ is direct as well, but presenting the resulting three-manifold pictorially without apparent self-intersections requires a little more care. The simplest way to achieve this is obtained by thinking of the manifold $\mathcal{N}_{\mathrm{X} ; q_{1} q_{2} \gamma \delta}$ of (3.9) as describing the three-manifold $D \times S^{1}$, with the disk $D$ lying in a horizontal plane and $S^{1}$ running vertically (that is, regarding $\mathrm{Y}_{\mathcal{T}}^{1}$ as part of the boundary of $\mathcal{N}_{\mathrm{X} ; q_{1} q_{2} \gamma \delta}$, rather than as a subset in the interior of $\mathcal{N}_{\mathrm{X} ; q_{1} q_{2} \gamma \delta}$ beyond which the three-manifold continues), and moreover regarding $D \times S^{1}$ as embedded in $S^{2} \times S^{1}$. One may then redraw (3.9) by exchanging interior and exterior 
regions, i.e. by representing the manifold $D \times S^{1}$ of (3.9) as the exterior of another copy of $D \times S^{1}$ in $S^{2} \times S^{1}$. This yields

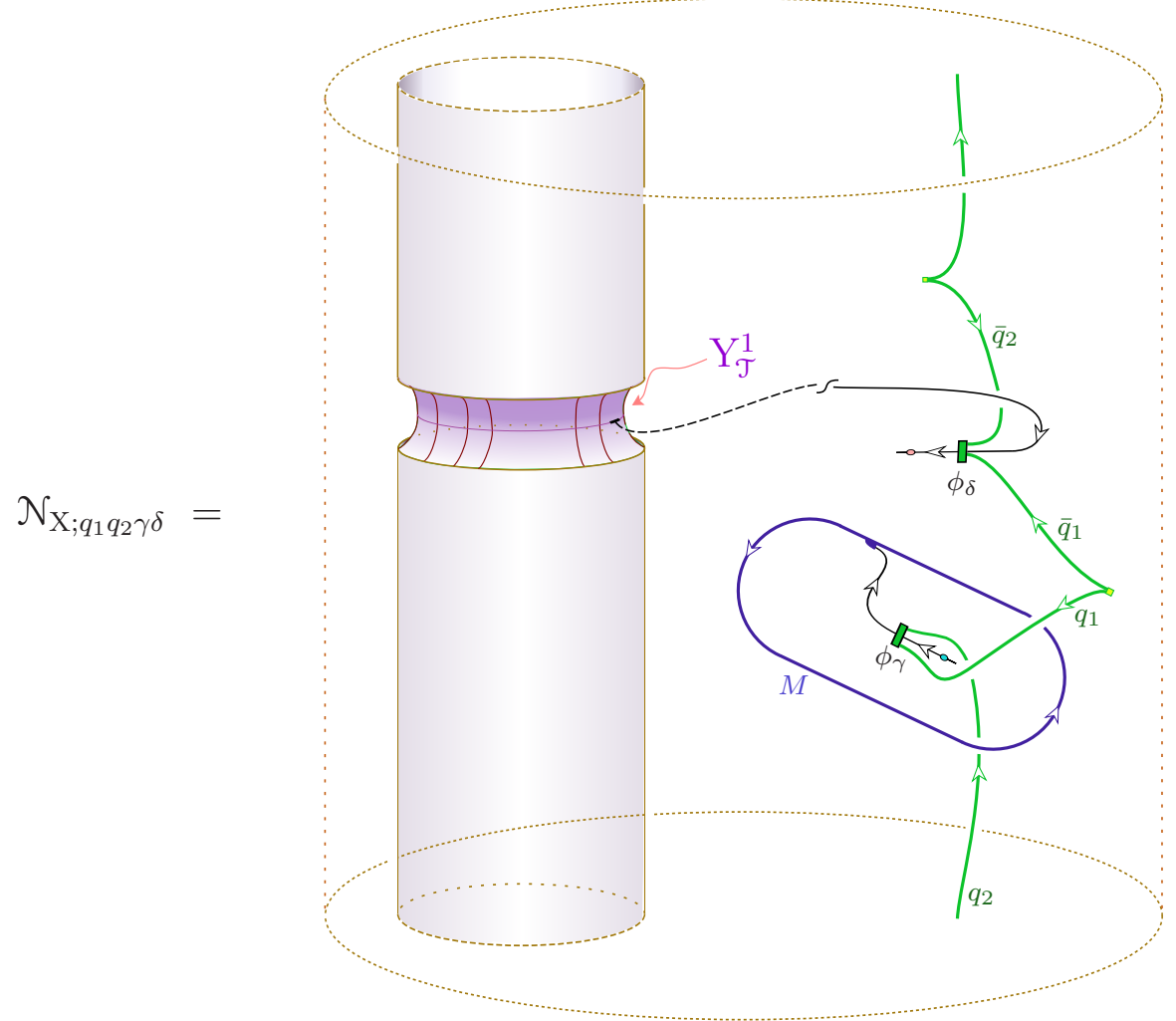

where the shaded region is the boundary, i.e. now the interior of the inner cylinder (that is, full torus, since top and bottom are identified) is the part not belonging to the manifold. Identification of $Y_{\mathcal{T}}^{1}$ in (3.10) with $\mathrm{Y}_{S}^{1}$ in (3.6) now yields the following description of the manifold $\mathcal{M}_{\mathrm{X} ; q_{1} q_{2} \gamma \delta}$ (from here on we restrict to the case that the number of bulk fields in the nibbled apple (3.6) is $n=2$ ):

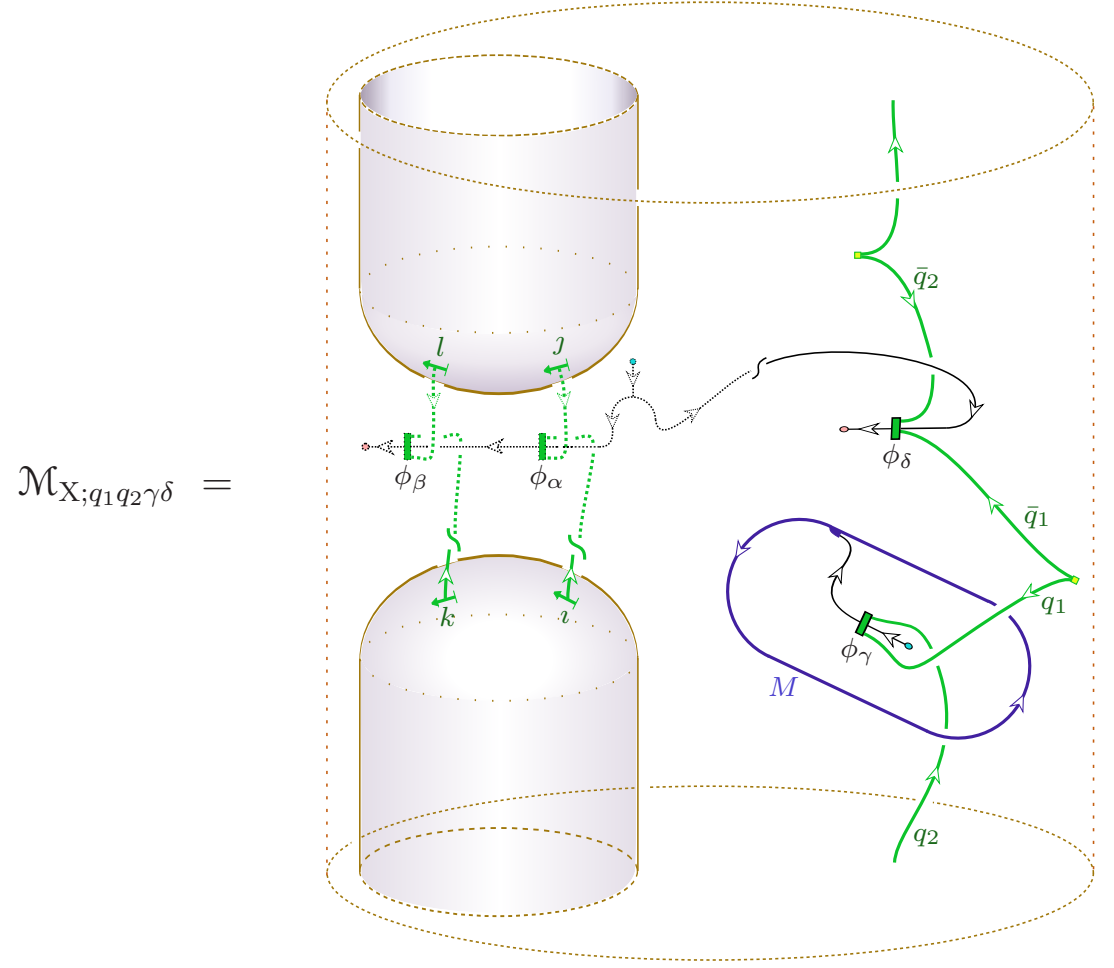


Note that the manifold $\mathcal{M}_{\mathrm{X} ; q_{1} q_{2} \gamma \delta}$ is not simply connected (and also has nonvtrivial second homotopy group); so even though its boundary is a two-sphere, $\mathcal{M}_{\mathrm{X} ; q_{1} q_{2} \gamma \delta}$ is not a three-ball. Further, part of the ribbon graph in (3.11) is drawn with dashed lines, which indicates that that part of the ribbons shows its 'black' side. To make it show its 'white' side we rotate this part of the ribbon graph by $\pi$ around a horizontal axis; this way $\mathcal{M}_{\mathrm{X} ; q_{1} q_{2} \gamma \delta}$ can be redrawn as

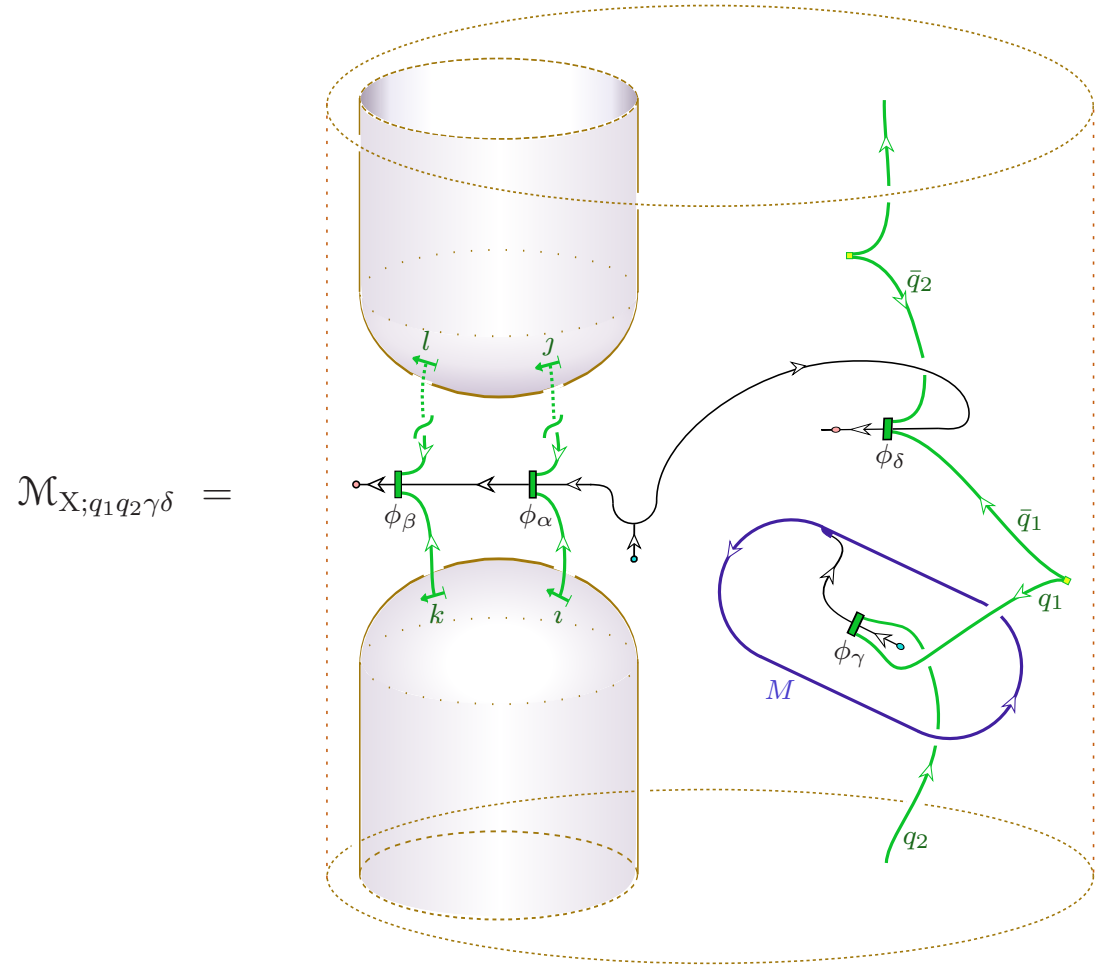

Analogously as in the case of boundary factorization, we are primarily interested in the contribution of the vacuum channel to the invariant $Z\left(\mathcal{M}_{\mathrm{X} ; q_{1} q_{2} \gamma \delta}\right)$. Extracting this contribution by applying the projetion $\pi_{0}$ we find

$$
c\left(\Phi_{\alpha}, \Phi_{\beta} ; M\right)_{0, \circ \circ}=\frac{1}{S_{0,0}} \sum_{q_{1}, q_{2} \in \mathcal{I}} \sum_{\gamma, \delta} \operatorname{dim}\left(U_{q_{1}}\right) \operatorname{dim}\left(U_{q_{2}}\right)\left(c_{q_{1}, q_{2}}^{\mathrm{bulk}}\right)_{\delta \gamma}^{-1} Z\left(\overline{\mathcal{M}}_{\mathrm{X} ; q_{1} q_{2} \gamma \delta}\right)
$$

where $\overline{\mathcal{M}}_{\mathrm{X} ; q_{1} q_{2} \gamma \delta}$ is the closed three-manifold $S^{2} \times S^{1}$ with an embedded ribbon graph as shown in 
the following picture:

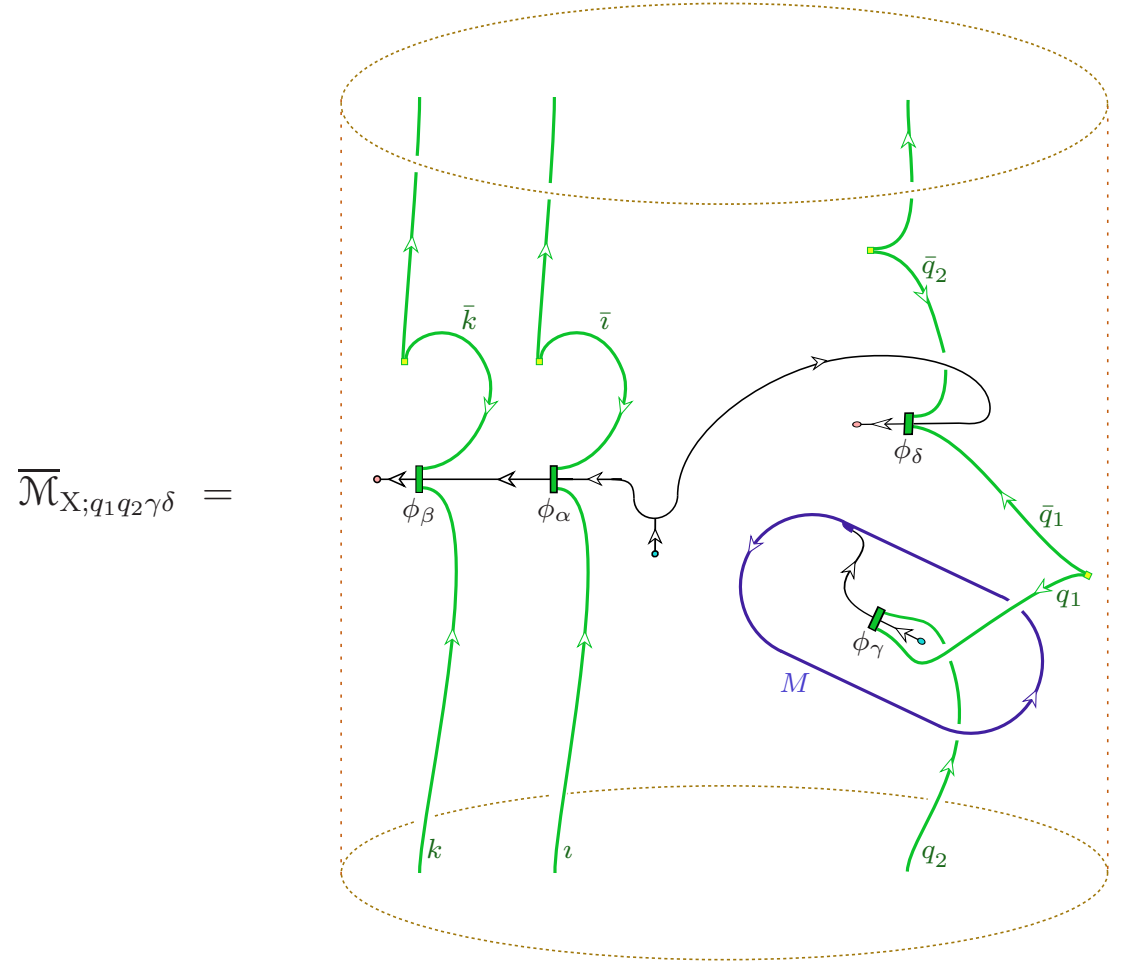

As one ingredient, (3.14) contains, in the lower right quarter of the picture, a morphism between the simple objects $U_{q_{2}}$ and $U_{\bar{q}_{1}}$. As a consequence, the number (3.14) is actually zero unless $q_{1}=\bar{q}_{2}=: q$. In the latter case, repeating the arguments relating the equalities (2.10) and (2.11), (3.14) can be rewritten as

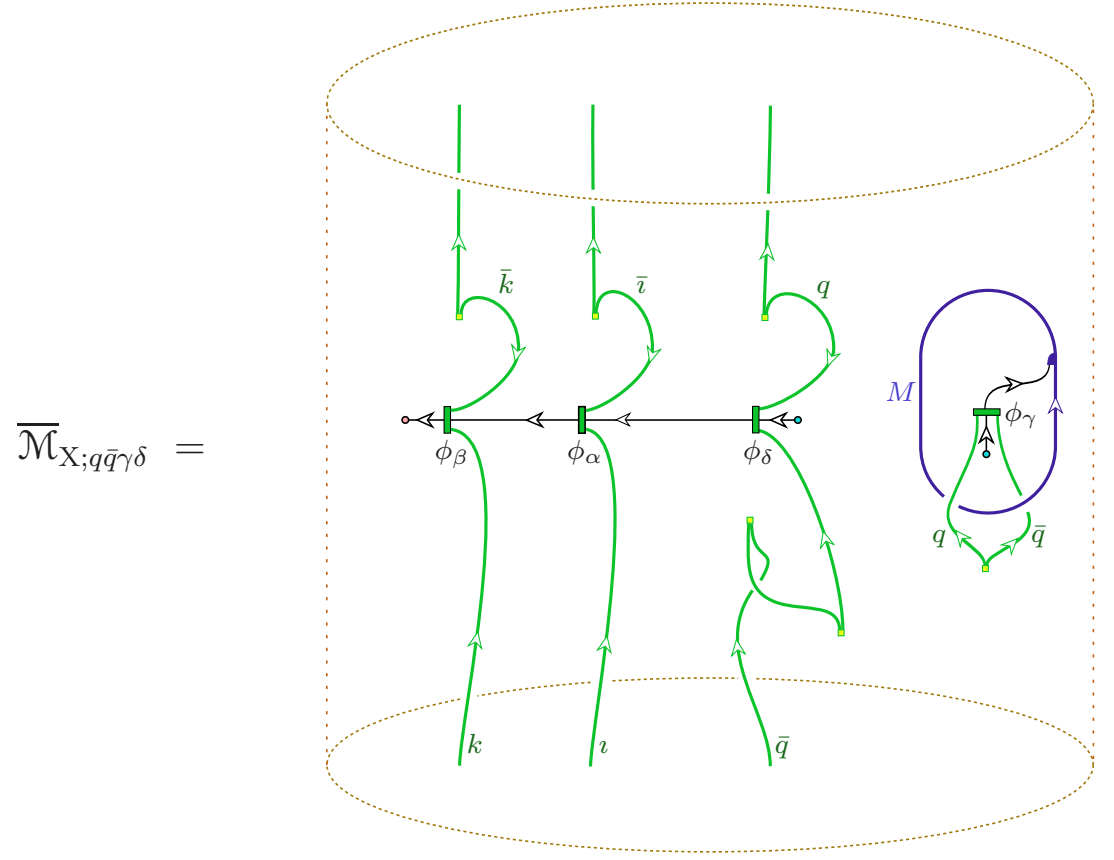

Now the disconnected part of the graph in (3.15) that contains the $M$-ribbon can be recognized as being nothing but the coefficient of the one-point function on the disk (compare figure (2.11) for $p=0)$. Moreover, in the disconnected part that contains the $U_{\imath^{-}}$and $U_{k}$-ribbons we can straighten 
out the $U_{\bar{q}}$-ribbon, which amounts to an additional factor of $\theta_{q} / \operatorname{dim}\left(U_{q}\right)$. Then (3.13) becomes

$$
c\left(\Phi_{\alpha}, \Phi_{\beta} ; M\right)_{0}=\frac{1}{S_{0,0}} \sum_{q \in \mathcal{I}} \sum_{\gamma, \delta} \theta_{q} \operatorname{dim}\left(U_{q}\right)\left(c_{q \bar{q}}^{\text {bulk }}\right)_{\delta \gamma}^{-1} K_{\beta \alpha \delta}^{k i \bar{q}} c\left(\Phi_{\gamma} ; M\right),
$$

with

$$
K_{\beta \alpha \delta}^{k \imath \bar{q}}=Z\left(\mathcal{K}_{\beta \alpha \delta}^{k \imath \bar{q}}\right)
$$

the invariant of the ribbon graph $\mathcal{K}_{\beta \alpha \delta}^{k \imath \bar{q}}$ shown in (1.2).

\section{The classifying algebra}

We now combine the results of the boundary and bulk factorizations. Expressing the equalities (2.16) and (3.16) in terms of the reflection coefficients $b_{M}^{\imath, \alpha}=b\left(\Phi_{\alpha} ; M\right)$ that we introduced in (2.19), we learn that

$$
b_{M}^{\imath, \alpha} b_{M}^{k, \beta}=\sum_{q \in \mathcal{I}} \sum_{\gamma=1}^{\mathrm{Z}_{q \bar{q}}} C_{\imath \alpha, k \beta}^{q \gamma} b_{M}^{q, \gamma}
$$

where $M$ is any elementary boundary condition and, for $\imath, \jmath, k \in \mathcal{I}$ and for $\alpha \in\left\{1,2, \ldots, \mathrm{Z}_{\imath \bar{\imath}}\right\}$ etc., the coefficients $C_{\imath \alpha, k \beta}^{q \gamma}$ are defined as

$$
C_{\imath \alpha, \jmath \beta}^{k \gamma}:=\frac{\theta_{k} \operatorname{dim}\left(U_{k}\right)}{S_{0,0}} \sum_{\delta=1}^{\mathrm{Z}_{k \bar{k}}}\left(c_{k \bar{k}}^{\mathrm{bulk}}\right)_{\delta \gamma}^{-1} K_{\beta \alpha \delta}^{\jmath \jmath \bar{k}}
$$

The following interpretation of the formula (4.1) suggests itself: In view of the description (A.10) of bulk fields, consider the complex vector space

$$
\mathcal{A}:=\bigoplus_{\imath \in \mathcal{I}} \operatorname{Hom}_{A \mid A}\left(U_{\imath} \otimes^{+} A \otimes^{-} U_{\bar{\imath}}, A\right)
$$

consisting of bulk fields which can have a non-vanishing one-point correlator on the disk. We endow this vector space in the following manner with the structure of an algebra. For each $\imath \in \mathcal{I}$ choose a basis $\left\{\phi^{\imath, \alpha} \mid \alpha=1,2, \ldots, \mathrm{Z}_{\imath}\right\}$ of $\operatorname{Hom}_{A \mid A}\left(U_{\imath} \otimes^{+} A \otimes^{-} U_{\bar{\imath}}, A\right)$. In this basis, we put the structure constants of the algebra $\mathcal{A}$ to be the numbers (4.2), i.e. we define the multiplication of $\mathcal{A}$ by

$$
\phi^{\imath, \alpha} \cdot \phi^{\jmath, \beta}:=\sum_{k, \gamma} C_{\imath \alpha, \jmath \beta}^{k \gamma} \phi^{k, \gamma} .
$$

Then for any elementary boundary condition $M$, the reflection coefficients $b_{M}^{\imath, \alpha}$ are the representation matrices, in the basis $\left\{\phi^{\imath, \alpha}\right\}$, for a one-dimensional representation $\rho_{M}$ of $\mathcal{A}$, i.e.

$$
b_{M}^{\imath, \alpha}=\rho_{M}\left(\phi^{\imath, \alpha}\right) .
$$

We will in fact show that $\mathcal{A}$ is a semisimple commutative associative algebra, so that every $\mathcal{A}$-representation is a direct sum of irreducible one-dimensional representations, and that the isomorphism classes of one-dimensional representations are in bijection with the inequivalent elementary boundary conditions. In particular, $\mathcal{A}$ plays indeed the role of a classifying algebra for boundary conditions - its representation theory determines the space of boundary conditions completely. 
We proceed to establish the relevant properties of $\mathcal{A}$. We first note that according to theorem 5.18 of [25] the dimension

$$
\operatorname{dim}_{\mathbb{C}}(\mathcal{A})=\sum_{\imath \in \mathcal{I}} \operatorname{dim}_{\mathbb{C}}\left(\operatorname{Hom}_{A \mid A}\left(U_{\imath} \otimes^{+} A \otimes^{-} U_{\bar{\imath}}, A\right)\right)=\sum_{\imath \in \mathcal{I}} \mathrm{Z}_{\imath \bar{\imath}}
$$

of $\mathcal{A}$ is equal to the number of inequivalent simple modules of the Frobenius algebra $A$. Moreover, the proof of this equality rests on the non-degeneracy of a certain matrix $\widetilde{S}^{A}$ (defined in (5.97) of [25]), and the entries of $\widetilde{S}^{A}$ are, in turn, proportional to the reflection coefficients $b_{M}^{\imath, \alpha}$, see e.g. formula (4.21) of [31]. This means in fact that, first, every simple $A$-module gives rise to a one-dimensional (and hence in particular irreducible) representation of $\mathcal{A}$, and second, in this way inequivalent simple $A$-modules give non-isomorphic $\mathcal{A}$-representations. It follows that the number $n_{\text {simp }}(\mathcal{A})$ of non-isomorphic irreducible $\mathcal{A}$-representations is at least as large as the number of inequivalent simple $A$-modules, and thus at least as large as the $\operatorname{dimension} \operatorname{dim}_{\mathbb{C}}(\mathcal{A})$ :

$$
n_{\operatorname{simp}}(\mathcal{A}) \geq \operatorname{dim}_{\mathbb{C}}(\mathcal{A})
$$

We will come back to this inequality below: once we have established associativity, we will see that the inequality is actually saturated. But for now, we switch to the proof of commutativity of $\mathcal{A}$, which turns out to be easy:

(A1) The algebra $\mathcal{A}$ is commutative.

Indeed, by merely using the defining properties of the symmetric special Frobenius algebra $A$ and the fact that the morphisms $\phi_{\alpha}$ and $\phi_{\beta}$ are morphisms of $A$-bimodules, it follows that the ribbon graph $\mathcal{K}_{\beta \alpha \delta}^{k v \bar{q}}$ can be transformed into the graph $\mathcal{K}_{\alpha \beta \delta}^{\imath k \bar{q}}$ without changing the value of the invariant. Thus $K_{\beta \alpha \delta}^{k v \bar{q}}=K_{\alpha \beta \delta}^{\imath k \bar{q}}$, which in turn implies that

$$
C_{\imath \alpha, k \beta}^{q \gamma}=C_{k \beta, \imath \alpha}^{q \gamma} .
$$

This argument is in fact nothing else than the one used to show that the correlators constructed according to the rules of 25 are independent of the choice of dual triangulation of the world sheet. It also has the virtue of showing at the same time that actually $K_{\beta \alpha \delta}^{k v \bar{q}}$ is totally symmetric in all three pairs $(k, \beta),(\imath, \alpha)$ and $(\bar{q}, \delta)$ of indices.

As a step towards further properties we express the structure constants (4.2) in a more accessible form. Consider the endomorphism that corresponds to the ribbon graph in the picture (1.2). By performing on this morphism two moves of the form displayed in (A.7), which each contribute an $\mathrm{F}[A]$-coefficient as a factor, one can rewrite $K_{\beta \alpha \delta}^{k i \bar{q}}$ as

$$
K_{\beta \alpha \delta}^{k \imath \bar{q}}=\operatorname{dim}(A) \sum_{\zeta, \eta_{1} \eta_{2}} \mathrm{~F}[A]_{\alpha \beta \zeta, q \bar{q} \eta_{1} \eta_{2}}^{(k \imath \overline{\bar{c}})} \mathrm{F}[A]_{\delta \zeta \circ, 00 \circ \circ}^{(q \bar{q} q \bar{q})} \varpi_{\eta_{1} \eta_{2}}^{k \imath \bar{q}}
$$


with $\varpi_{\eta_{1} \eta_{2}}^{k \imath \bar{q}}$ the morphism

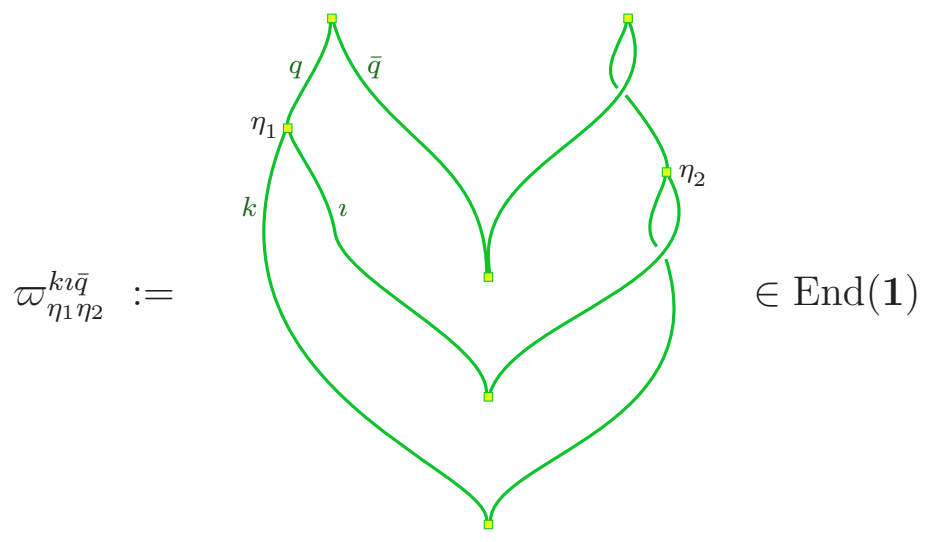

When inserted into (4.2), together with (A.16) it follows that the structure constants can be written as

$$
C_{\imath \alpha, k \beta}^{q \gamma}=\operatorname{dim}\left(U_{q}\right) \theta_{q} \sum_{\eta_{1}, \eta_{2}} \mathrm{~F}[A]_{\alpha \beta \gamma, q \bar{q} \eta_{1} \eta_{2}}^{(k \imath \overline{\bar{k}})} \varpi_{\eta_{1} \eta_{2}}^{k \imath \bar{q}} .
$$

Further it is easy to see that when one of the three bulk fields, say $\Phi_{\delta}$, involved in $K_{\beta \alpha \delta}^{k i \bar{q}}$ is the identity field, we have

$$
K_{\beta \alpha}^{k \imath} \equiv K_{\beta \alpha \circ}^{k \imath 0}=\delta_{\bar{k}, \imath} \operatorname{dim}(A) \mathrm{F}[A]_{\alpha \beta \circ, 00 \circ \circ}^{(\bar{\imath} \imath \bar{\imath})} \mathrm{R}_{\circ \circ}^{(\bar{\imath} \imath) 0} \mathrm{~F}_{\circ 0 \circ, \circ 0 \circ}^{(\bar{\imath} \imath) \imath}
$$

where the last factor is a fusing coefficient as defined in $(A .3)$ and $R_{\circ \circ}^{(\bar{\imath} \imath) 0}$ is a braiding coefficient. With the help of the identities (A.16) and (see (2.2.46) of [25]) $\mathbf{R}_{\circ \circ}^{(\bar{\imath} \imath) 0} \mathrm{~F}_{\circ 0 \circ, 00 \circ}^{(i \bar{i} i) i}=\left(\theta_{i} \operatorname{dim}\left(U_{i}\right)\right)^{-1}$, this can be rewritten as

$$
K_{\beta \alpha \circ}^{k \imath 0}=\delta_{\bar{k}, \imath} \frac{S_{0,0}}{\theta_{\imath} \operatorname{dim}\left(U_{\imath}\right)} c_{\bar{\imath} \imath, \beta \alpha}^{\text {bulk }} .
$$

We can now conclude:

\section{(A2) The algebra $\mathcal{A}$ is unital.}

Indeed, using (4.13) together with the total symmetry of $K_{\beta \alpha \delta}^{k i \bar{q}}$ in its three pairs of indices one checks that

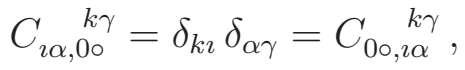

i.e. the element $t^{0 \circ} \in \mathcal{A}$, which corresponds to the identity field, is a unit for the product of the algebra $\mathcal{A}$.

The proof of associativity turns out to be harder. Note in particular that associativity is not manifest when the structure constants are written in the form (4.11). A crucial observation is instead that our arguments leading to the formula (4.1) generalize to the case of a correlator of a general number $n$ (instead of just 2) of bulk fields. Rather than leading to an ordinary binary product, one then obtains an $n$-ary product on the vector space $\mathcal{A}$, and the structure constants for this product in the basis $\left\{t^{\imath \alpha}\right\}$ are given by

$$
C_{\imath_{1} \alpha_{1}, l_{2} \alpha_{2}, \ldots, l_{n} \alpha_{n}}^{k \gamma}=\frac{\theta_{k} \operatorname{dim}\left(U_{k}\right)}{S_{0,0}} \sum_{\delta}\left(c_{k \bar{k}}^{\mathrm{bulk}}\right)_{\delta \gamma}^{-1} K_{\alpha_{n} \alpha_{n-1} \ldots \alpha_{1} \delta}^{l_{n} \imath_{n-1} \ldots \imath_{1} \bar{k}}
$$


with $K_{\alpha_{n} \alpha_{n-1} \ldots \alpha_{1} \delta}^{\imath_{n} \imath_{n-1} \ldots \imath_{1} \bar{k}}$ the invariant of the ribbon graph

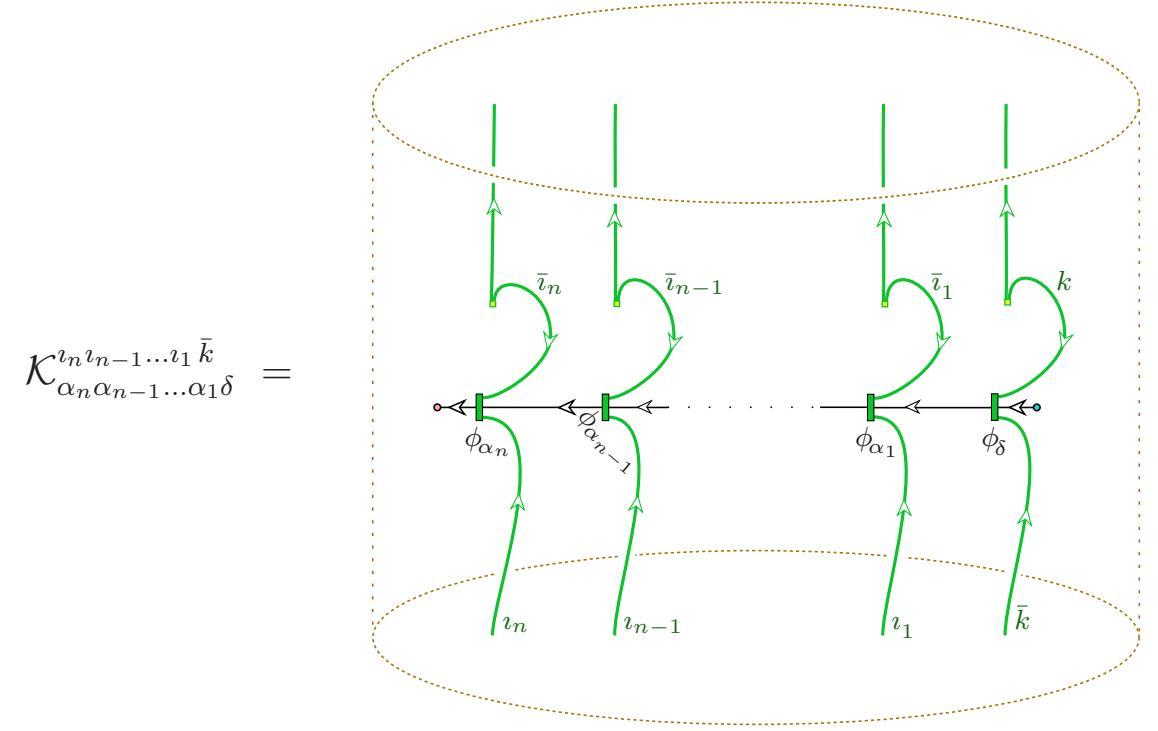

in $S^{2} \times S^{1}$. Note that by the same argument as in the case of the binary product, the structure constants of the $n$-ary products are totally symmetric in all pairs of lower indices. In other words, the $n$-ary products are totally commutative.

The numbers (4.16) can again be expressed in terms of $\mathrm{F}[A]$-coefficients. In particular, for the case $n=3$ we find

$$
K_{\gamma \beta \alpha \delta}^{k \imath \jmath \bar{q}}=\sum_{l_{1}, l_{2}, m_{1}, m_{2}, p} \sum_{\substack{\mu, \nu_{1}, \nu_{2}, \rho, \sigma_{1}, \sigma_{2}, \tau_{1}, \tau_{2}}} \mathrm{~F}[A]_{\beta \gamma \mu, l_{1} l_{2} \nu_{1} \nu_{2}}^{(k \jmath \bar{k} \bar{k})} \mathrm{F}[A]_{\alpha \mu \rho, m_{1} m_{2} \sigma_{1} \sigma_{2}}^{\left(l_{1} i \bar{\imath} \ell_{2}\right)} \mathrm{F}[A]_{\delta \rho \circ, p \bar{p} \tau_{1} \tau_{2}}^{\left(m_{1} \bar{q} q m_{2}\right)} L_{\mu \nu_{1} \nu_{2} \rho \sigma_{1} \sigma_{2} \tau_{1} \tau_{2}}^{k \imath \bar{q} ; l_{1} l_{2} m_{1} m_{2} p}
$$

with $L_{\mu \nu_{1} \nu_{2} \rho \sigma_{1} \sigma_{2} \tau_{1} \tau_{2}}^{k \imath \jmath} l_{1} l_{2} l_{2} m_{1} m_{2} p$ invariant of the ribbon graph

$\mathcal{L}_{\mu \nu_{1} \nu_{2} \rho \sigma_{1} \sigma_{2} \tau_{1} \tau_{2}}^{k \imath \bar{q} ; l_{1} l_{2} m_{1} m_{2} p}:=$

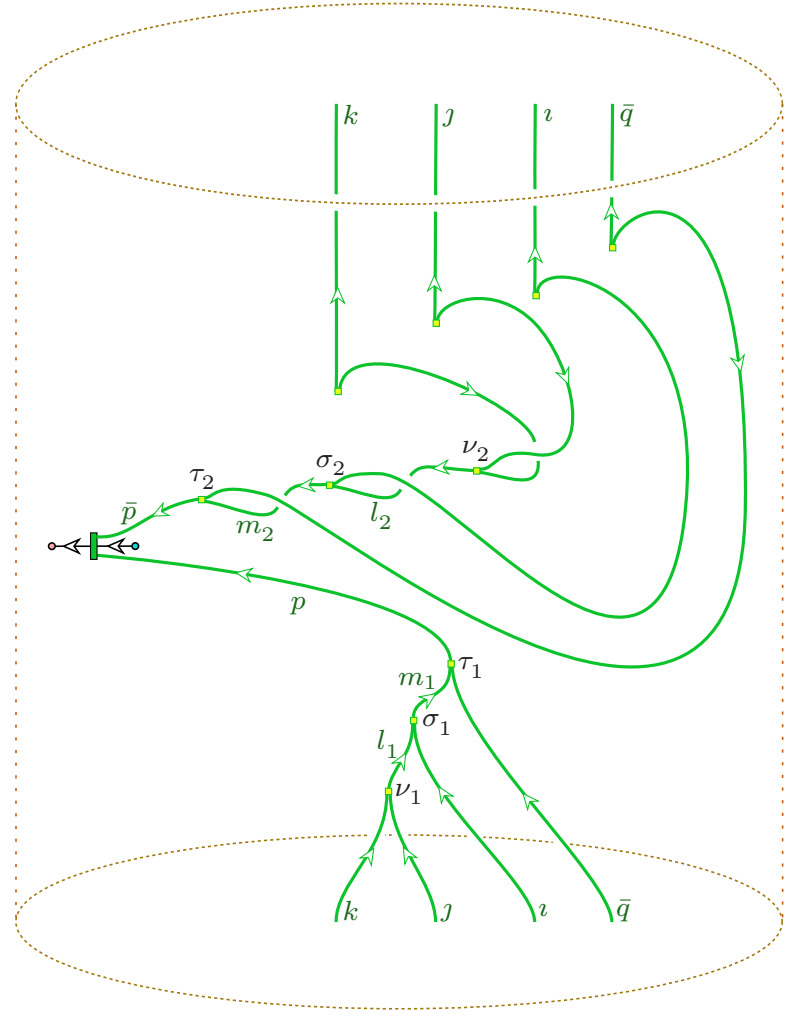


in $S^{2} \times S^{1}$. This invariant vanishes unless $p=0$, in which case (4.17) simplifies to (using also (A.16)

$$
K_{\gamma \beta \alpha \delta}^{k \imath \jmath \bar{q}}=S_{0,0} \sum_{l_{1}, l_{2}} \sum_{\substack{\mu, \nu_{1}, \nu_{2}, \rho, \sigma_{1}, \sigma_{2}}} \mathrm{~F}[A]_{\beta \gamma \mu, l_{1} l_{2} \nu_{1} \nu_{2}}^{(k j \bar{k})} \mathrm{F}[A]_{\alpha \mu \rho, q \bar{q} \sigma_{1} \sigma_{2}}^{\left(l_{1} i \bar{\ell} \ell_{2}\right)} c_{q \bar{q}, \rho \delta}^{\mathrm{bulk}} \tilde{\varpi}_{\nu_{1} \nu_{2}, \sigma_{1} \sigma_{2}}^{k j i \bar{q}, l_{1} l_{2}}
$$

with $\tilde{\varpi}_{\nu_{1} \nu_{2}, \sigma_{1} \sigma_{2}}^{k j z \bar{l} l_{1} l_{2}}$ the morphism

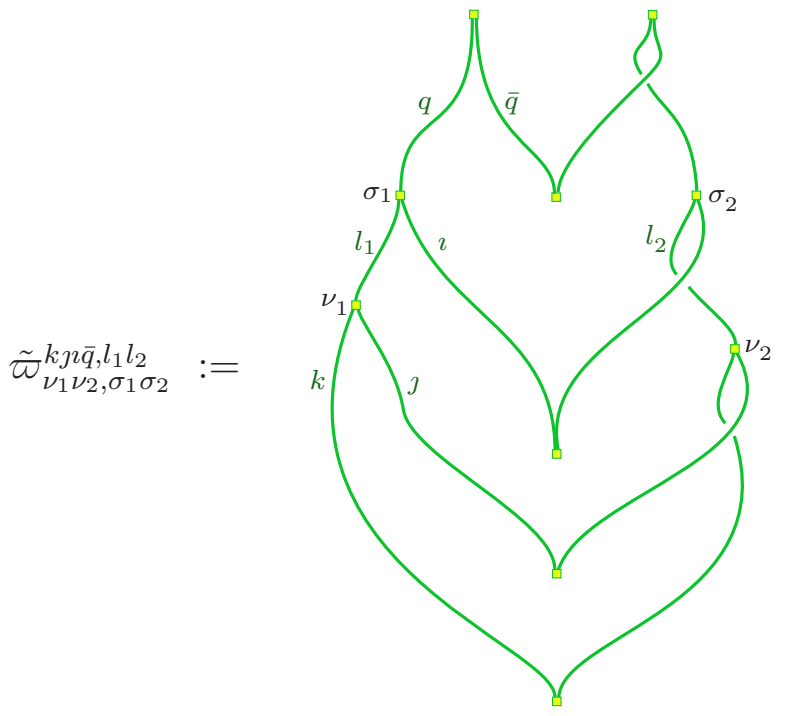

After using dominance in $\operatorname{Hom}\left(U_{l_{1}} \otimes U_{l_{2}}, U_{l_{1}} \otimes U_{l_{2}}\right)$, only the contribution from the tensor unit survives in the resulting summation. As a consequence (4.20) can be factorized as

$$
\tilde{\varpi}_{\nu_{1} \nu_{2}, \sigma_{1} \sigma_{2}}^{k j i \bar{q} l_{1} l_{2}}=\delta_{l_{2}, \bar{l}_{1}} \operatorname{dim}\left(U_{l_{1}}\right) \theta_{l_{1}} \varpi_{\sigma_{1} \sigma_{2}}^{l_{1} r \bar{q}} \varpi_{\nu_{1} \nu_{2}}^{k j \bar{l}_{1}}
$$

with the factors $\varpi$ as defined in (4.10).

We now insert this result into (4.15) and also perform the $\delta$-summation; this yields the structure constants of the ternary product as

$$
C_{\imath \alpha, \jmath \beta, k \gamma}^{q \delta}=\operatorname{dim}\left(U_{q}\right) \theta_{q} \sum_{l \in \mathcal{I}} \sum_{\mu, \nu_{1}, \nu_{2}, \sigma_{1}, \sigma_{2}} \operatorname{dim}\left(U_{l}\right) \theta_{l} \mathrm{~F}[A]_{\beta \gamma \mu, l \bar{l} \nu_{1} \nu_{2}}^{(k J \bar{k} \bar{k})} \mathrm{F}[A]_{\alpha \mu \delta, q \bar{q} \sigma_{1} \sigma_{2}}^{(l \imath \bar{l} \bar{l})} \varpi_{\sigma_{1} \sigma_{2}}^{l l \bar{q}} \varpi_{\nu_{1} \nu_{2}}^{k j \bar{l}}
$$

Thus by comparison with (4.11) it follows that

$$
C_{\imath \alpha, \jmath \beta, k \gamma}^{q \delta}=\sum_{\ell, \mu} C_{\jmath \beta, k \gamma}^{\ell \mu} C_{\imath \alpha, \ell \mu}^{q \delta}
$$

On the other hand, using in addition the total commutativity of the products, one also has

$$
C_{\imath \alpha, \jmath \beta, k \gamma}^{q \delta}=C_{k \gamma, \jmath \beta, \imath \alpha}^{q \delta}=\sum_{\ell, \mu} C_{\jmath \beta, \imath \alpha}^{\ell \mu} C_{k \gamma, \ell \mu}^{q \delta}=\sum_{\ell, \mu} C_{\imath \alpha, \jmath \beta}^{\ell \mu} C_{\ell \mu, k \gamma}^{q \delta} .
$$

Together, (4.23) and (4.24) establish:

(A3) The algebra $\mathcal{A}$ is associative.

Once we know that $\mathcal{A}$ is associative, we are finally in a position to address the issue of semisimplicity. Namely, as any finite-dimensional associative algebra, $\mathcal{A}$ is isomorphic, as an $\mathcal{A}$-module, to a direct sum of all inequivalent indecomposable projective $\mathcal{A}$-modules, each one occuring with 
a multiplicity given by the dimension of the corresponding simple $\mathcal{A}$-module (see e.g. Satz G.10 of [36]). Comparing dimensions, this tells us in particular that the $\operatorname{dimension}_{\operatorname{dim}}(\mathcal{C})$ is at least as large as the number $n_{\text {simp }}(\mathcal{A})$ of non-isomorphic irreducible $\mathcal{A}$-representations. In view of the opposite inequality (4.7), this means that these two numbers are atually equal,

$$
n_{\text {simp }}(\mathcal{A})=\operatorname{dim}_{\mathbb{C}}(\mathcal{A}) .
$$

This is only possible if atually each of these irreducible representations is one-dimensional as well as projective, and thus $\mathcal{A}$ is isomorphic to the direct sum of the corresponding one-dimensional $\mathcal{A}$-modules. In particular this means:

(A4) The algebra $\mathcal{A}$ is semisimple.

Thus we have established the claim that the classifying algebra $\mathcal{A}$ is a semisimple commutative associative algebra.

Let us also add the following observations:

- When the Frobenius algebra $A$ is Morita equivalent to the tensor unit $\mathbf{1}$ - the so-called Cardy case - the expression (4.3) for $\mathcal{A}$ reduces to $\bigoplus_{\imath \in \mathcal{I}} \operatorname{Hom}\left(U_{\imath} \otimes U_{\bar{\imath}}, \mathbf{1}\right)=\bigoplus_{\imath \in \mathcal{I}} \mathbb{C}$, and the structure constants become the fusion rules, $C_{\imath, j}{ }^{k}=\operatorname{dim}_{\mathbb{C}} \operatorname{Hom}\left(U_{\imath} \otimes U_{\jmath} \otimes U_{k}, \mathbf{1}\right)$. Thus in this case the classifying algebra is nothing else than the Verlinde algebra $K_{0}(\mathcal{C}) \otimes_{\mathbb{Z}} \mathbb{C}$ of the category $\mathcal{C}$. In particular, it then has a canonical basis.

- The structure constants furnish the matrix elements of the regular representation of $\mathcal{A}$. Since $\mathcal{A}$ is semisimple, the regular representation is fully reducible, hence its representation matrices can be diagonalized simultaneously.

- By (4.2) and (4.13) we have

$$
C_{\imath \alpha, \jmath \beta}^{0 \circ}=\frac{1}{S_{0,0}}\left(c_{00}^{\mathrm{bulk}}\right)^{-1} K_{\beta \alpha \circ}^{\jmath \imath 0}=\left[\theta_{\imath} \operatorname{dim}\left(U_{\imath}\right) c_{00}^{\mathrm{bulk}}\right]^{-1} \delta_{\bar{\jmath}, \imath} c_{\imath \bar{\imath}, \alpha \beta}^{\mathrm{bulk}} .
$$

Thus by the non-degeneracy of the bulk two-point functions on the sphere these structure constants provide a non-degenerate bilinear form on $\mathcal{A}$. This allows us to introduce structure constants with only lower indices:

$$
C_{\imath \alpha, \jmath \beta, k \gamma}:=\sum_{\ell, \delta} C_{\imath \alpha, \jmath \beta}^{\ell \delta} C_{\ell \delta, k \gamma}^{0 \circ} .
$$

Inserting the expressions (4.2), (4.26) and (A.17), these are simply given by

$$
C_{\imath \alpha, \jmath \beta, k \gamma}=\frac{1}{\operatorname{dim}(A)} K_{\beta \alpha \gamma}^{\jmath \imath k} .
$$

In particular, they are totally symmetric in the three pairs of indices.

Also note that by associativity of $\mathcal{A}$ the bilinear form is invariant, while by commutativity it is symmetric. Thus $\mathcal{A}$ has the structure of a symmetric Frobenius algebra.

- In the same way as in (4.23) above it follows that for any $n$-ary product and any $\ell=1,2, \ldots, n-1$ an analogous factorization of the $n$-ary product into $(\ell+1)$ - and $(n-\ell+1)$-ary products holds, and all sequences of concatenations of arbitrary products are compatible. Thus the products defined by (4.15) provide a representation of the associative operad.

With the previous results at hand, we are finally also in a position to discuss the compatibility with the mapping class group representations on the spaces of conformal blocks on the sphere. 
The mapping class group $G_{n}$ of the $n$-punctured sphere is generated by braiding homeomorphisms which interchange any two insertion points and by Dehn twists around each insertion point. Now consider the ribbon graph $\widehat{\mathcal{K}}$ shown in figure (1.2), or rather its generalization $\widehat{\mathcal{K}}_{n}$ to the case of any number $n$ of bulk field insertions. The invariant $\widehat{K}_{n}=Z\left(\widehat{\mathcal{K}}_{n}\right)$ of this ribbon graph constitutes an endomorphism of the space of $n$-point conformal blocks on the sphere.

Now one can easily convince oneself that commuting any generator of $G_{n}$, acting on the horizontal factor $S^{2}$ of the three-manifold, through $\widehat{\mathcal{K}}_{n}$ merely amounts to a change of the dual triangulation of $S^{2}$ that underlies the horizontal part of the ribbon graph. By the independence from the choice of dual triangulation of the world sheet as already invoked above, it therefore follows that the invariant $\widehat{K}_{n}$ commutes with the action of $G_{n}$ on the space of conformal blocks, i.e. that $\widehat{K}_{n}$ intertwines the action of the mapping class group on the space of blocks. This argument is similar to the one showing invariance of the correlators under the mapping class group, compare section 3 of [30].

Acknowledgments: We thank Valya Petkova for urging us to use the results of the TFT approach to RCFT correlators to revamp our understanding of the classifying algebra.

JF is partially supported by VR under project no. 621-2006-3343. CSc is partially supported by the Collaborative Research Centre 676 "Particles, Strings and the Early Universe - the Structure of Matter and Space-Time", and would like to thank Karlstad University for hospitality. CSt gratefully acknowledges a Kähler research fellowship from the Center for Mathematical Physics Hamburg. 


\section{A Appendix}

\section{A.1 Chiral data}

The chiral symmetries of a CFT can be realized in the structure of a conformal vertex algebra. From a conformal vertex algebra one can, in turn, construct (see e.g. [37]) a chiral CFT. Crucial information about the chiral theory is contained in the representation category $\mathcal{C}$ of the vertex algebra. For a rational $\mathrm{CFT} \mathcal{C}$ is, by definition, a (semisimple) modular tensor category.

Despite its name, a chiral CFT is not a conventional quantum field theory: instead of local correlation functions it supplies a system of conformal blocks, i.e. vector bundles with connection over the moduli spaces of curves with marked points. A chiral CFT can, however, be used as an ingredient in the construction of a full local CFT. For these quantum field theories, many aspects, including the computation of structure constants of operator product expansions, can be discussed on the basis of $\mathcal{C}$ as an abstract category (which can in particular be taken to be strict, i.e. with strictly associative tensor product and with tensoring by the tensor unit $\mathbf{1}$ acting as the identity on objects), without reference to its concrete realization as the representation category of a vertex algebra.

A modular tensor category is in particular semisimple and $\mathbb{C}$-linear and has a finite number of isomorphism classes of simple objects. We denote representatives for these classes by $U_{i}$, with $i$ taking values in a finite index set $\mathcal{I}$, and choose $\mathcal{I} \ni 0$ such that $U_{0}=\mathbf{1}$ is the tensor unit. For any simple object $U$ its space of endomorphisms is isomorphic to $\mathcal{C}, \operatorname{End}(U) \equiv \operatorname{Hom}(U, U)=\mathbb{C i d}_{U}$; we identify the space $\operatorname{End}(\mathbf{1}, \mathbf{1})=\mathbb{C i d}_{\mathbf{1}}$ with $\mathbb{C}$. The (quantum) dimension of an object $V$ is denoted by $\operatorname{dim}(V)$.

As $\mathcal{C}$ is semisimple and $\mathbb{C}$-linear, any morphism between objects $V$ and $W$ of $\mathcal{C}$ can be written as a sum of morphisms between simple subobjects of $V$ and of $W$; this property is known as dominance. Also, the essential properties of the tensor product are already captured by the spaces of morphisms between tensor products of simple objects. In particular we may express any morphism space as a direct sum of tensor products (over $\mathbb{C}$ ) of spaces of three-point couplings of simple objects, i.e. of the morphism spaces $\operatorname{Hom}\left(U_{i} \otimes U_{j}, U_{k}\right)$ and their duals $\operatorname{Hom}\left(U_{k}, U_{i} \otimes U_{j}\right)$ with $i, j, k \in \mathcal{I}$. This reduces the choice of bases in arbitrary morphism spaces to the choice of bases of all such spaces of three-point couplings. We select bases $\left\{\lambda_{i j}^{k \alpha}\right\}$ of all spaces $\operatorname{Hom}\left(U_{i} \otimes U_{j}, U_{k}\right)$ and keep them fixed throughout the paper; for the dual spaces $\operatorname{Hom}\left(U_{k}, U_{i} \otimes U_{j}\right)$ we choose the dual bases. We depict these bases as

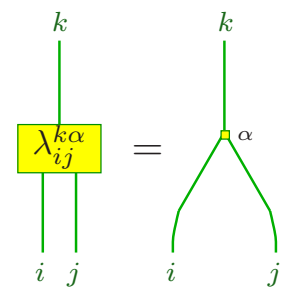

and

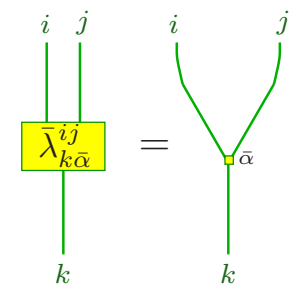

Lines labeled by $0 \in \mathcal{I}$ can be omitted, as they correspond to the tensor unit $U_{0}=\mathbf{1}$. Further, taking $\bar{k}$ to be the unique element of $\mathcal{I}$ for which $U_{\bar{k}}$ is isomorphic to $U_{k}^{\vee}$, we abbreviate

$$
\lambda_{i \bar{\imath}} \equiv \lambda_{i \bar{\imath}}^{0 \circ}=\bigwedge_{\imath} \text { and } \quad \bar{\lambda}^{\imath \bar{\imath}} \equiv \bar{\lambda}_{0 \circ}^{\imath \bar{\imath}}=\left.\right|^{\imath}
$$


In this case the spaces are one-dimensional, so that the label takes only a single value, for which we choose the symbol ' $\circ$ '. Moreover, just like in the pictures just displayed we often suppress this label in our notations.

When expressing the bases of morphism spaces involving multiple tensor products in terms of the chosen bases of three-point couplings, several choices are possible, corresponding to the different possible orders in which to perform the tensor products. The two possibilities for a triple tensor product are related by a fusing move, according to

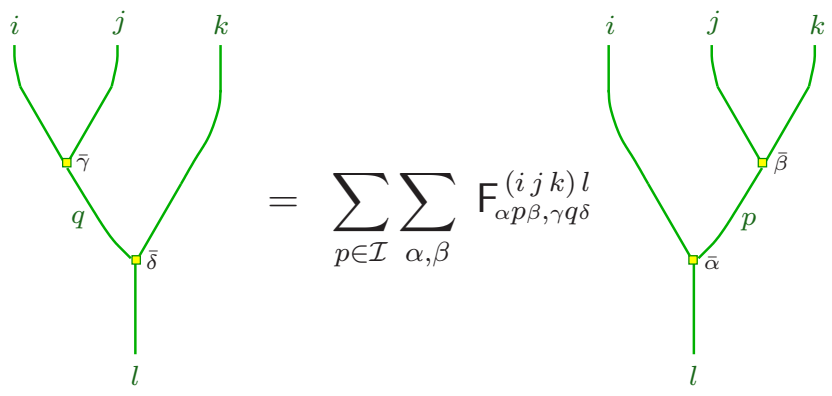

The fusing matrices $\mathrm{F}$ satisfy a pentagon identity, assuring that any two different orderings of performing any multiple tensor product are related by a suitable sequence of fusing moves.

To specify a standard basis of the space $B\left(V_{1}, \ldots, V_{m}\right)$ of $m$-point chiral blocks on the sphere, with insertions labeled by objects $V_{1}, V_{2}, \ldots, V_{m}$ of $\mathcal{C}$, we note that this space is isomorphic to the space $\operatorname{Hom}\left(\mathbf{1}, V_{1} \otimes V_{2} \otimes \cdots \otimes V_{m}\right)$ of morphisms of $\mathcal{C}$. And in accordance with the comments about dominance made above we decompose the latter space as

$$
\begin{array}{r}
\operatorname{Hom}\left(\mathbf{1}, V_{1} \otimes V_{2} \otimes \cdots \otimes V_{m}\right)= \\
\otimes_{p_{0}, p_{1}, p_{2}, \ldots, p_{m-3} \in \mathcal{I}}\left[\operatorname{Hom}\left(\mathbf{1}, V_{1} \otimes U_{p_{0}}\right) \otimes_{\mathbb{C}} \operatorname{Hom}\left(U_{p_{0}}, V_{2} \otimes U_{p_{1}}\right)\right. \\
\left.\otimes_{\mathbb{C}} \operatorname{Hom}\left(U_{p_{m-3}}, V_{m-1} \otimes U_{p_{2}}\right) \otimes_{\mathbb{C}} \cdots \otimes_{\mathbb{C}} \operatorname{Hom}\left(U_{p_{m-4}}, V_{m-2} \otimes U_{p_{m-3}}\right)\right] .
\end{array}
$$

Taking the objects $V_{k}=U_{\imath_{k}}$ for $k=1,2, \ldots, m$ to be simple, a basis of $B\left(U_{\imath_{1}}, \ldots, U_{\imath_{m}}\right)$ corresponding to this decomposition is given by the elements (2.6).

\section{A.2 Algebras in tensor categories}

Besides the category $\mathcal{C}$, the construction of a full CFT needs one other piece of algebraic data: an object $A$ of $\mathcal{C}$ endowed with the structure of a symmetric special Frobenius algebra. Here by the term algebra one means a unital associative algebra; in complete analogy with a conventional algebra in the category of vector spaces, an object of a tensor category is an algebra iff there exist a multiplication morphism $m \in \operatorname{Hom}(A \otimes A, A))$ and a unit morphism $\eta \in \operatorname{Hom}(\mathbf{1}, A)$ obeying associativity and unitality relations, respectively. An algebra is Frobenius iff it is also a coalgebra, with the coproduct being an isomorphism of $A$-bimodules. For a Frobenius algebra $A$ there exist two distinguished isomorphisms between $A$ and its dual $A^{\vee} ; A$ is symmetric iff these two isomorphisms are equal. (Equivalently, a Frobenius algebra may be characterized by the categorical analogue of a nondegenerate invariant bilinear form; then the algebra is symmetric iff that form is symmetric, see e.g. [38.) A Frobenius algebra is special iff the counit is a left-inverse of the unit and the product is a left-inverse of the coproduct. For more details about algebras in $\mathcal{C}$ see e.g. section 3.1 of [25]. 
Modules and bimodules over an algebra in a tensor category can again be studied in full analogy with the classical case of algebras in the category of vector spaces. In the case of a modular tensor category $\mathcal{C}$, the braiding of $\mathcal{C}$ can be used to obtain, for any object $U$ of $\mathcal{C}$, two $A$-bimodule structures $U \otimes^{ \pm} A$ and $A \otimes^{ \pm} U$ on the objects $U \otimes A$ and $A \otimes U$, respectively. For details see e.g. sections 4.1 and 5.4 of [25].

The dimensions

$$
\mathrm{Z}_{p q}:=\operatorname{dim}_{\mathbb{C}}\left(\operatorname{Hom}_{A \mid A}\left(U_{p} \otimes^{+} A \otimes^{-} U_{q}, A\right)\right)
$$

of the morphism space $\operatorname{Hom}_{A \mid A}\left(U_{p} \otimes^{+} A \otimes^{-} U_{q}, A\right)$ involving such specific bimodules give the entries of the matrix $Z$ that describes the torus partition function $Z$ in the standard basis of characters. Also, for such spaces of bimodule morphisms we have isomorphisms

$$
\begin{aligned}
& \operatorname{Hom}_{A \mid A}\left(U_{j} \otimes^{+} A \otimes^{-} U_{k}, A\right) \otimes_{\mathbb{C}} \operatorname{Hom}_{A \mid A}\left(U_{i} \otimes^{+} A \otimes^{-} U_{l}, A\right) \\
& \stackrel{\cong}{\longrightarrow} \bigoplus_{q, q^{\prime} \in \mathcal{I}} \operatorname{Hom}\left(U_{i} \otimes U_{j}, U_{q}\right) \otimes_{\mathbb{C}} \operatorname{Hom}\left(U_{k} \otimes U_{l}, U_{q^{\prime}}\right) \otimes_{\mathbb{C}} \operatorname{Hom}_{A \mid A}\left(U_{q} \otimes^{+} A \otimes^{-} U_{q^{\prime}}, A\right) .
\end{aligned}
$$

We denote the coefficients of these isomorphisms with respect to a standard choice of bases by $\mathrm{F}[A]_{\alpha \beta \gamma, q q^{\prime} \delta \delta^{\prime}}^{(i j k l)}$, i.e.

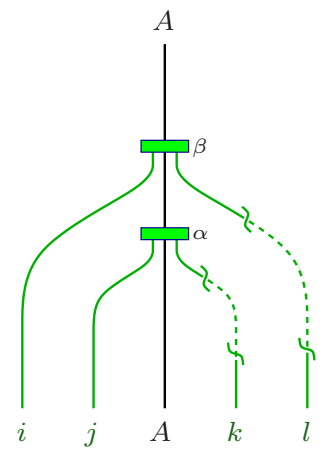

$$
=\sum_{q, q^{\prime} \in \mathcal{I}} \sum_{\gamma=1}^{\mathrm{Z}_{q q^{\prime}}} \sum_{\delta=1}^{N_{i j}{ }^{q}} \sum_{\delta^{\prime}=1}^{N_{i g^{q^{\prime}}}} \mathrm{F}[A]_{\alpha \beta \gamma, q q^{\prime} \delta \delta^{\prime}}^{(i j k l)}
$$

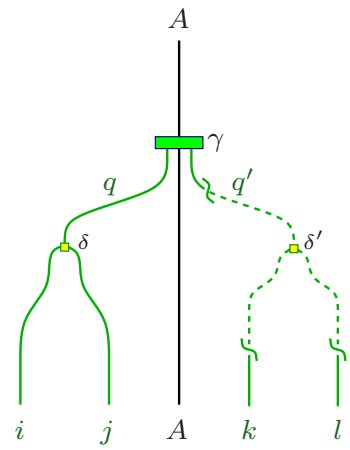

These coefficients are special cases (namely for all involved defect lines $X$ being the trivial defect line $X_{0}=A$ ) of the entries of fusion matrices for defect fields, see section 2.2 of [31].

\section{A.3 Three-dimensional topological field theory}

For studying rational chiral CFT, tools from three-dimensional topological quantum field theory

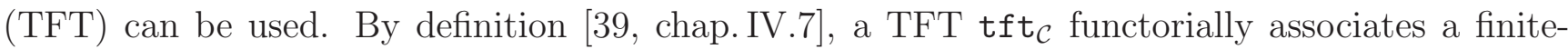
dimensional vector space $\operatorname{tft}_{\mathcal{C}}(E)$ to any extended surface $E$, and a linear map from $\operatorname{tft}_{\mathcal{C}}(E)$ to $\operatorname{tft}_{\mathcal{C}}\left(E^{\prime}\right)$ to any extended cobordism $\mathcal{M}: E \rightarrow E^{\prime}$; the image $\operatorname{tft}_{\mathcal{C}}(\mathcal{M})$ is also called the invariant of $\mathcal{M}$ and denoted by $Z(\mathcal{M})$. The surfaces $E$ and cobordisms $\mathcal{M}$ form the objects and morphisms of the decorated cobordism category $\mathcal{C}_{\mathrm{C}}$. The modular tensor category $\mathcal{C}$ provides decoration data for $\mathcal{C o b}_{\mathcal{C}}$. In more detail, an extended surface $E$ is, roughly, a compact closed oriented twomanifold with a finite set of embedded arcs, each arc being marked by an object of $\mathcal{C}$. An extended cobordism $E \rightarrow E^{\prime}$ is a compact oriented three-manifold $\mathcal{M}$ satisfying $\partial \mathcal{M}=(-E) \sqcup E^{\prime}$, together with an oriented ribbon graph $\Gamma_{\mathcal{M}}$ in $\mathcal{M}$ such that at each marked arc of $(-E) \sqcup E^{\prime}$ a ribbon of $\Gamma_{\mathcal{N}}$ is ending. Each ribbon of $\Gamma_{\mathcal{M}}$ is labeled by an object of $\mathcal{C}$, while each coupon of $\Gamma_{\mathcal{M}}$ is labeled by an element of the morphism space of $\mathcal{C}$ that corresponds to the objects of the ribbons which enter and leave the coupon. 


\section{A.4 The TFT construction of full CFT}

The construction of full CFTs using tools from TFT, for brevity to be called the TFT construction, has been accomplished in [25, 31, 30]. Here we summarize some pertinent features of the construction. (We suppress various ingredients, such as the role of the orientation of ribbons or of boundary components, and restrict our attention to oriented world sheets. For a few more details see section 4.3 of [40]; a comprehensive exposition can e.g. be found in appendix B of [30].)

While a full CFT is defined on a world sheet X - a surface with a conformal structure, which may in particular have non-empty boundary - the corresponding chiral CFT is defined on the complex double $\widehat{X}$ of $X$, i.e. the orientation bundle over $\mathrm{X}$ modulo identification of the two points in the fibre over each boundary point of $X$. The double $\widehat{X}$ can be endowed with the structure of an extended surface. X can be obtained from its double $\widehat{\mathrm{X}}$ as the quotient by an orientation-reversing involution $\tau$. In particular, the double of the disk is the two-sphere, with $\tau$ acting, in standard complex coordinates, as $z \mapsto \bar{z}^{-1}$.

Further, when X comes with field insertions, that is, embedded arcs labeled either by objects of $\mathcal{C}$ (for boundary fields, corresponding to $\operatorname{arcs}$ on $\partial \mathrm{X}$ ) or by pairs of objects of $\mathcal{C}$ (for bulk fields, corresponding to arcs in the interior of $\mathrm{X}$ ), then there are corresponding arcs on $\widehat{\mathrm{X}}$ labeled by objects of $\mathcal{C}$. A correlation function $c_{\mathrm{X}}$ of the full $\mathrm{CFT}$ on $\mathrm{X}$ is a specific element in the resulting space of conformal blocks of the chiral CFT on the double $\widehat{X}$. The TFT construction gives this element as

$$
c_{\mathrm{X}}=\operatorname{tft}_{\mathcal{C}}\left(\mathcal{M}_{\mathrm{X}}\right) 1 \in \operatorname{tft}_{\mathcal{C}}(\widehat{\mathrm{X}})
$$

with $1 \in \mathbb{C}=\operatorname{tft}_{\mathcal{C}}(\emptyset)$.

Here $\mathcal{M}_{\mathrm{X}} \equiv \emptyset \stackrel{\mathcal{M}_{\mathrm{X}}}{\longrightarrow} \widehat{\mathrm{X}}$, called the connecting manifold for the world sheet $\mathrm{X}$, is an extended cobordism constructed from the data of $\mathrm{X}$, the category $\mathcal{C}$, and a symmetric special Frobenius algebra $A$ in $\mathcal{C}$. $\mathcal{M}_{\mathrm{X}}$ is assembled as follows.

- As an oriented three-manifold, $\mathcal{M}_{\mathrm{X}}$ is the interval bundle over $\mathrm{X}$ modulo a $\mathbb{Z}_{2}$-identification of the intervals over $\partial \mathrm{X}: \mathcal{M}_{\mathrm{X}}=(\widehat{\mathrm{X}} \times[-1,1]) / \sim$ with $([x$, or $], t) \sim([x,-$ or $],-t)$, where or denotes the local 2-orientation. It follows in particular that $\partial \mathcal{M}_{\mathrm{X}}=\widehat{\mathrm{X}}$ and that $\mathrm{X}$ is naturally embedded in $\mathcal{M}_{\mathrm{X}}$ as $\mathrm{I}: \mathrm{X} \stackrel{\simeq}{\rightarrow} \mathrm{X} \times\{t=0\} \hookrightarrow \mathcal{M}_{\mathrm{X}}$. The fiber in $\mathcal{M}_{\mathrm{X}}$ over a point of $\mathrm{X}$ is called a connecting interval.

Compare picture (1.1), in which the connecting intervals are displayed for the case of the disk.

- Contained in $\mathcal{M}_{\mathrm{X}}$ there is a the ribbon graph $\Gamma_{\mathcal{M}_{\mathrm{X}}} \subset \mathcal{M}_{\mathrm{X}}$. A crucial part of $\Gamma_{\mathcal{N}_{\mathrm{X}}}$ is a (dual) oriented triangulation $\Gamma$ of $\mathrm{I}(\mathrm{X})$. The edges and vertices of $\Gamma \backslash \mathrm{I}(\partial \mathrm{X})$ are labeled by objects and morphisms of $\mathcal{C}$, respectively, and it is here that the Frobenius algebra $A$ enters: an edge of $\Gamma \backslash \mathrm{I}(\partial \mathrm{X})$ is covered with a ribbon labeled by the object $A$, while each (three-valent) vertex is covered with a coupon labeled by the multiplication morphism $m$ of $A .2$

That $c_{\mathrm{X}}$ does not depend on the choice of triangulation $\Gamma$ is equivalent to the assertion that the object $A$ carries the structure of a symmetric special Frobenius algebra.

- If X has non-empty boundary, each edge $e$ of $\Gamma \cap \mathrm{I}(\partial \mathrm{X})$ is covered with a ribbon labeled by a left $A$-module $N=N(e)$, while each vertex of $\Gamma$ lying on $\mathrm{I}(\partial \mathrm{X})$ is covered with a coupon labeled by the representation morphism $\rho_{N} \in \operatorname{Hom}(A \otimes N, N)$. The $A$-module $N$ formalizes the notion of the boundary condition associated to a segment of $\partial \mathrm{X}$.

\footnotetext{
2 Standing alone, these assignments can be in conflict with the orientations of some of the edges of $\Gamma$. This conflict is resolved by inserting along each such edge a coupon with a suitable morphism, in either $\operatorname{Hom}(A \otimes A, \mathbf{1})$ or $\operatorname{Hom}(\mathbf{1}, A \otimes A)$, coming from the invariant bilinear form of $A$.
} 
- Bulk and boundary fields are implemented by coupons lying in the interior, respectively covering a boundary arc, of $\mathrm{I}(\mathrm{X})$, which are labeled by appropriate morphisms. These are described in more detail in the next subsection.

In the picture (2.3) the cobordism resulting from this description is shown for the case of a disk with $n$ bulk field insertions.

The correlators (A.8) obtained by the prescription above satisfy [30] all consistency constraints. Conversely, for a CFT obeying some natural non-degeneracy assumptions, any consistent set of correlators can be obtained through the TFT construction [26].

When displaying ribbon graphs arising in the TFT construction, for simplicity we draw ribbons just as lines or, rather, framed lines, with blackboard framing being implicit. Occasionally the ribbon graphs also involve half-twists of ribbons, which change the visible side of a ribbon from its 'white' to its 'black' side or vice versa. In blackboard framing, the white and black sides of a ribbon are indicated by a solid and a dashed line, respectively; see e.g. the picture (A.7) above.

In the process of calculating invariants of ribbon graphs, one projects the graphs in a nonsingular manner to a plane and interprets the resulting pictures as morphisms of the category $\mathcal{C}$. These resulting morphisms can in particular involve the duality, braiding and twist morphisms of $\mathcal{C}$; for these we employ the pictorial conventions summarized e.g. in appendix A.1 of [30].

\section{A.5 Bulk and boundary fields}

As explained in detail in section 3.2 .1 of [31]), a boundary field insertion $\Psi$ can be characterized as

$$
\Psi=(M, N, V, \psi, p,[\gamma])
$$

by the following data: two boundary conditions (i.e. left $A$-modules) $M$ and $N$, an object $V$ of the category $\mathcal{C}$ of chiral data, a morphism $\psi$ in the space $\operatorname{Hom}_{A}(M \otimes V, N)$ of module morphisms, a point $p$ on the boundary $\partial \mathrm{X}$ of the world sheet, and finally a germ $[\gamma]$ of arcs aligned to $\partial \mathrm{X}$ at $\gamma(0)=p$. Also, for every component of $\partial \mathrm{X}$ an orientation must be specified. All correlators can be obtained from a set of basic correlators for which $V=U_{i}$ is a simple object and $\psi=\psi_{\alpha}$ is a basis element of the morphism space $\operatorname{Hom}_{A}\left(M \otimes U_{i}, N\right)$.

Similarly, as described in e.g. section 3.3.1 of [31] (and in accordance with formula (A.5) a bulk field insertion

$$
\Phi=\left(V, V^{\prime}, \phi, p,\left[\gamma_{\phi}\right], \text { or }\right)
$$

is determined by the following data: two objects $V$ and $V^{\prime}$ of $\mathcal{C}$, a bimodule morphism $\phi$ in $\operatorname{Hom}_{A \mid A}\left(V \otimes^{+} A \otimes^{-} V^{\prime}, A\right)$, a point $p$ in the interior of $\mathrm{X}$, a germ $[\gamma]$ of arcs at $p$, and a local orientation or of $\mathrm{X}$ around $p$. One can obtain all correlators from those which involve bulk fields for which $V=U_{i}$ and $V^{\prime}=U_{j}$ are simple objects and $\phi=\phi_{\alpha}$ is a basis element of the bimodule morphism space $\operatorname{Hom}_{A \mid A}\left(U_{i} \otimes^{+} A \otimes^{-} U_{j}, A\right)$.

In addition, both for boundary fields and for bulk fields one must divide out a certain equivalence relation (see definitions 3.3 and 3.6 of [31]). In the main text the insertion points, arc-germs and local orientations are suppressed, as they do not play a direct role in our considerations. Moreover, to reduce the notational burden, we usually suppress the boundary labels $M, N$ as well as the chiral labels $i, j, \ldots \in \mathcal{I}$ when they can be recovered from the rest of the information. In particular we write

$$
\Psi_{\alpha} \equiv \Psi_{\alpha}^{M N ; j}=\left(M, N, U_{j}, \psi_{\alpha}\right) \quad \text { and } \quad \Phi_{\alpha} \equiv \Phi_{\alpha}^{i j}=\left(U_{i}, U_{j}, \phi_{\alpha}\right)
$$

for boundary and bulk fields, respectively. 


\section{A.6 Two-point functions}

The standard two-point correlation function of boundary fields on the unit disk with boundary segments carrying boundary conditions $M$ and $N$, as defined e.g. in formula (2.22) of [30], is an element in the space of two-point chiral blocks on the sphere. We consider the case that the chiral insertions are given by simple objects and, correspondingly, select chiral labels $k$ and $l=\bar{k}$ for the boundary fields, so that this space of blocks is one-dimensional (for $l \neq \bar{k}$ the space is zero). For given boundary fields, the coefficient of the two-point function with respect to a basis in the one-dimensional space of blocks is denoted by $c_{M, N, k ; \psi^{+}, \psi^{-}}^{\text {bnd }}$, where the morphisms $\psi^{ \pm}$specify the boundary fields in question. Choosing bases $\left\{\psi_{\alpha}^{+}\right\}$and $\left\{\psi_{\beta}^{-}\right\}$in the spaces $\operatorname{Hom}_{A}\left(M \otimes U_{k}, N\right)$ and $\operatorname{Hom}_{A}\left(N \otimes U_{\bar{k}}, M\right)$ of boundary fields with fixed chiral labels, these numbers are arranged into a square matrix with entries

$$
\left(c_{M, N, k}^{\mathrm{bnd}}\right)_{\alpha \beta}:=c_{M, N, k ; \psi_{\alpha}^{+}, \psi_{\beta}^{-}}^{\mathrm{bnd}} .
$$

As shown in appendix C.1 of [30], for any fixed values of $M, N$ and $k$ this matrix furnishes a non-degenerate bilinear form $\operatorname{Hom}_{A}\left(M \otimes U_{k}, N\right) \times \operatorname{Hom}_{A}\left(N \otimes U_{\bar{k}}, M\right) \rightarrow \operatorname{Hom}\left(U_{k} \otimes U_{\bar{k}}, \mathbf{1}\right)$.

In the particular case that $M=N$ is a simple $A$-module and $k=0$, the space $\operatorname{Hom}_{A}\left(M \otimes U_{0}, M\right)$ has the unit constraint as a canonical basis. In this basis, we denote the value of the bilinear form by

$$
\left(c_{M, M, 0}^{\mathrm{bnd}}\right)_{\text {○० }}^{-1}=: c_{M, 0}^{\mathrm{bnd}} .
$$

As one sees directly from formula (C.3) of [30, we have

$$
c_{M, 0}^{\mathrm{bnd}}=\operatorname{dim}(M)
$$

The standard two-point function of bulk fields on the sphere, as defined e.g. in formula (2.41) of [30, is an element in the tensor product of two copies of the space of two-point chiral blocks on the sphere. We assume that the chiral labels of the two bulk fields $\Phi$ and $\Phi^{\prime}$ are conjugate to each other, say $\Phi=\Phi_{\imath \jmath}, \Phi^{\prime}=\Phi_{\bar{\imath} \bar{\jmath}}$, in which case this space is one-dimensional (while it is zero otherwise). With this choice of chiral labels, the coefficient of the two-point function with respect to a basis of the space of blocks is denoted by $c_{i, j, \phi, \phi^{\prime}}^{\text {bulk }}$. Selecting bases $\left\{\phi_{\alpha}\right\}$ of $\operatorname{Hom}_{A \mid A}\left(U_{i} \otimes^{+} A \otimes^{-} U_{j}, A\right)$ and $\left\{\phi_{\beta}^{\prime}\right\}$ of $\operatorname{Hom}_{A \mid A}\left(U_{\bar{\imath}} \otimes^{+} A \otimes^{-} U_{\bar{\jmath}}, A\right)$ for the relevant spaces of bimodule morphisms, the coefficients arrange into a matrix $c_{i, j}^{\text {bulk }}$ by

$$
c_{i, j, \phi_{\alpha}, \phi_{\beta}^{\prime}}^{\text {bulk }}=:\left(c_{i, j}^{\text {bulk }}\right)_{\alpha \beta} .
$$

For fixed $i, j$ this furnishes a non-degenerate bilinear form $\operatorname{Hom}_{A \mid A}\left(U_{i} \otimes^{+} A \otimes^{-} U_{j}, A\right) \times \operatorname{Hom}_{A \mid A}$ $\left(U_{\bar{\imath}} \otimes^{+} A \otimes^{-} U_{\bar{\jmath}}, A\right) \rightarrow \operatorname{Hom}\left(U_{\imath} \otimes U_{\bar{\imath}}, \mathbf{1}\right) \otimes_{\mathbb{C}} \operatorname{Hom}\left(U_{\jmath} \otimes U_{\bar{\jmath}}, \mathbf{1}\right)$, see appendix C.2 of [30].

A graphical description of the numbers $\left(c_{i, j}^{\text {bulk }}\right)_{\alpha \beta}$ is given in formula (C.14) of [30]. This actually constitutes one instance in which the coefficients $\mathrm{F}[A]$ introduced in (A.7) occur naturally. Namely, by performing an $\mathrm{F}[A]$-move on the expression (C.14) of [30] one finds

$$
c_{q \bar{q}, \alpha \beta}^{\mathrm{bulk}}=\frac{\operatorname{dim}(A)}{S_{0,0}} \mathrm{~F}[A]_{\beta \alpha \circ, 00 \circ \circ}^{(q \bar{q} q \bar{q})} .
$$

Analogously as for boundary fields, there exists a canonical basis in the case that the bulk field is the tensor unit, i.e. $q=0$. In this basis we have

$$
c_{00}^{\text {bulk }}=\frac{\operatorname{dim}(A)}{S_{0,0}} .
$$




\section{References}

[1] H. Sonoda, Sewing conformal field theories, Nucl. Phys. B 311 (1988) 401

[2] D.C. Lewellen, Sewing constraints for conformal field theories on surfaces with boundaries, Nucl. Phys. B 372 (1992) 654

[3] G. Pradisi, A. Sagnotti, and Ya.S. Stanev, Completeness conditions for boundary operators in 2D conformal field theory, Phys. Lett. B 381 (1996) 97 [hep-th/9603097]

[4] A.A. Belavin, A.M. Polyakov, and A.B. Zamolodchikov, Infinite conformal symmetry in two-dimensional quantum field theory, Nucl. Phys. B 241 (1984) 333

[5] R.E. Behrend, P.A. Pearce, V.B. Petkova, and J.-B. Zuber, Boundary conditions in rational conformal field theories, Nucl. Phys. B 579 (2000) 707 [hep-th/9908036]

[6] G. Felder, J. Fröhlich, J. Fuchs, and C. Schweigert, The geometry of WZW branes, J. Geom. and Phys. 34 (2000) 162 [hep-th/9909030]

[7] I. Affleck and A.W.W. Ludwig, Universal noninteger "ground-state degeneracy" in critical quantum systems, Phys. Rev. Lett. 67 (1991) 161

[8] I. Brunner, M.R. Douglas, A. Lawrence, and C. Römelsberger, D-Branes on the quintic, J. High Energy Phys. 0008 (2000) 015 [hep-th/9906200]

[9] J. Fuchs and C. Schweigert, A classifying algebra for boundary conditions, Phys. Lett. B 414 (1997) 251 [hep-th/9708141]

[10] V. Pasquier, Operator content of ADE lattice models, J. Phys. A 20 (1987) 5707

[11] C. Schweigert and J. Fuchs, Classifying algebras for boundary conditions and traces on spaces of conformal blocks, in: Theory of Elementary Particles, H. Dorn et al., eds. (Wiley-VCH, Berlin 1998), p. 13

[12] J. Fuchs and C. Schweigert, The action of outer automorphisms on bundles of chiral blocks, Commun. Math. Phys. 206 (1999) 691 [hep-th/9805026]

[13] J. Fuchs and C. Schweigert, Orbifold analysis of broken bulk symmetries, Phys. Lett. B 447 (1999) 266 [hep-th/9811211]

[14] J. Fuchs and C. Schweigert, Symmetry breaking boundaries I. General theory, Nucl. Phys. B 558 (1999) 419 [hep-th/9902132]

[15] L. Birke, J. Fuchs, and C. Schweigert, Symmetry breaking boundary conditions and WZW orbifolds, Adv. Theor. Math. Phys. 3 (1999) 671 [hep-th/9905038]

[16] J. Fuchs, L.R. Huiszoon, A.N. Schellekens, C. Schweigert, and J. Walcher, Boundaries, crosscaps and simple currents, Phys. Lett. B 495 (2000) 427 [hep-th/0007174]

[17] M.R. Gaberdiel, A. Recknagel, and G.M.T. Watts, The conformal boundary states for SU(2) at level 1, Nucl. Phys. B 626 (2002) 344 [hep-th/0108102]

[18] T.P.T. Dijkstra, L.R. Huiszoon and A.N. Schellekens, Supersymmetric standard model spectra from RCFT orientifolds, Nucl. Phys. B 710 (2005) 3 [hep-th/0411129]

[19] K. Hosomichi, Permutation orientifolds of Gepner models, J. High Energy Phys. 0701 (2007) 081 [hep-th/0612109]

[20] L.E. Ibáñez, A.N. Schellekens, and A.M. Uranga, Instanton induced neutrino Majorana masses in CFT orientifolds with MSSM-like spectra, J. High Energy Phys. 0706 (2007) 011 [0704.1079]

[21] E.B. Kiritsis, A.N. Schellekens, and M. Tsulaia, Discriminating MSSM families in (free-field) Gepner orientifolds, J. High Energy Phys. 0810 (2008) 106 [0809.0083]

[22] G. Moore and N. Seiberg, Classical and quantum conformal field theory, Commun. Math. Phys. 123 (1989) 177

[23] B. Bakalov and A.N. Kirillov, On the Lego-Teichmüller game, Transform. Groups 5 (2000) 207 [math.GT/9809057] 
[24] J. Fuchs, I. Runkel, and C. Schweigert, Conformal correlation functions, Frobenius algebras and triangulations, Nucl. Phys. B 624 (2002) 452 [hep-th/0110133]

[25] J. Fuchs, I. Runkel, and C. Schweigert, TFT construction of RCFT correlators I: Partition functions, Nucl. Phys. B 646 (2002) 353 [hep-th/0204148]

[26] J. Fjelstad, J. Fuchs, I. Runkel, and C. Schweigert, Uniqueness of open/closed rational CFT with given algebra of open states, Adv. Theor. Math. Phys. 12 (2008) 1281 [hep-th/0612306]

[27] L. Kong, Cardy condition for open-closed field algebras, Commun. Math. Phys. 283 (2008) 25 [math.QA/0612255]

[28] L. Kong and I. Runkel, Morita classes of algebras in modular tensor categories, Adv. in Math. 219 (2008) 1548 [math.CT/0708.1897]

[29] N.Yu. Reshetikhin and V.G. Turaev, Invariants of 3-manifolds via link polynomials and quantum groups, Invent. math. 103 (1991) 547

[30] J. Fjelstad, J. Fuchs, I. Runkel, and C. Schweigert, TFT construction of RCFT correlators V: Proof of modular invariance and factorisation, Theory and Appl. Cat. 16 (2006) 342 [hep-th/0503194]

[31] J. Fuchs, I. Runkel, and C. Schweigert, TFT construction of RCFT correlators IV: Structure constants and correlation functions, Nucl. Phys. B 715 (2005) 539 [hep-th/0412290]

[32] J.E. Andersen and J. Fjelstad, Reducibility of quantum representations of mapping class groups, preprint math.QA/0806.2539

[33] J.E. Andersen and J. Fjelstad, On reducibility of mapping class group representations: The $S U(N)$ case, preprint math.QA/0902.4375

[34] J.L. Cardy and D.C. Lewellen, Bulk and boundary operators in conformal field theory, Phys. Lett. B 259 (1991) 274

[35] J. Fuchs and C. Schweigert, Branes: from free fields to general backgrounds, Nucl. Phys. B 530 (1998) 99 [hep-th/9712257]

[36] J.C. Jantzen and J. Schwermer, Algebra (Springer Verlag, Berlin 2005)

[37] E. Frenkel and D. Ben-Zvi, Vertex Algebras and Algebraic Curves, second edition (American Mathematical Society, Providence 2004)

[38] J. Fuchs and C. Stigner, On Frobenius algebras in rigid monoidal categories, Arabian Journal for Science and Engineering 33-2C (2008) 175 [hep-th/0901.4886]

[39] V.G. Turaev, Quantum Invariants of Knots and 3-Manifolds (de Gruyter, New York 1994)

[40] J. Fuchs, T. Nikolaus, C. Schweigert, and K. Waldorf, Bundle gerbes and surface holonomy, preprint math.DG/0901.2085; Proceedings of the 5th European Congress of Mathematics, in press 